Review

\title{
Prevention of Graphene Restacking for Performance Boost of Supercapacitors-A Review
}

\section{Jiantong Li * and Mikael Östling}

KTH Royal Institute of Technology, School of Information and Communication Technology, Electrum 229, Kista SE-16440, Sweden; E-Mail: ostling@kth.se

* Author to whom correspondence should be addressed; E-Mail: jiantong@kth.se; Tel.: +46-8-790-4342; Fax: +46-8-790-4300.

Received: 6 December 2012; in revised form: 6 February 2013 / Accepted: 16 February 2013 / Published: 6 March 2013

\begin{abstract}
Graphene is a promising electrode material for supercapacitors mainly because of its large specific surface area and high conductivity. In practice, however, several fabrication issues need refinement. The restacking of graphene flakes upon being packed into supercapacitor electrodes has become a critical challenge in the full utilization of graphene's large specific surface area to further improve the device performance. In this review, a variety of recent techniques and strategies are overviewed for the prevention of graphene restacking. They have been classified into several categories to improve and facilitate the discussion on the underlying ideas. Based on the overview of the existing techniques, we discuss the trends of future research in the fields.
\end{abstract}

Keywords: graphene; supercapacitor; restacking; spacer; conducting polymer; carbon nanotube; pseudo-capacitive metal oxide; ternary; three dimensional (3D) network; hierarchical structure

\section{Introduction}

Energy storage systems have been playing an important role in our lives. There are a great deal of applications, including portable electronics, hybrid electric vehicles and large industrial equipment [1]. At present, batteries are the most widely used systems which may store energy at densities as high as $180 \mathrm{Wh} / \mathrm{kg}[1,2]$. However, batteries suffer from many limitations, such as low power density (only 50-200 W/kg), long charging time $(0.3-3 \mathrm{~h})$, limited cycle life, abrupt failure, poor low-temperature 
kinetics, and safety concerns caused by the usage of lithium [3,4]. In many applications, supercapacitors are expected to complement or even replace batteries. Supercapacitors, also called electrochemical capacitors or ultracapacitors, typically comprise two electrodes immersed in an electrolyte with a thin layer of separator in between, and two current collectors (metal) connecting the electrodes [3]. They store energy using the simple charge separation at the electrochemical interface (double layer) between the high-surface-area electrode and the electrolyte, and/or using pseudo-capacitance resulting from fast surface redox reactions. Consequently, supercapacitors are often of high power density (1-10 kW/kg), short charge/discharge time (in seconds), and long life cycles (over $10^{5}$ times). As compared with batteries, the main shortcoming for supercapacitors is their relatively low energy density $(1-10 \mathrm{Wh} / \mathrm{kg})$. Further improvement of their performance mainly relies on advanced electrode materials, which should possess high electrical conductivity, large specific surface area, and long-term electrochemical stability [3].

Graphene, a flat monolayer of carbon atoms tightly packed into a two-dimensional (2D) honeycomb lattice [5], has high electrical conductivity (minimally of the order of $4 e^{2} / h[5,6]$ ), large specific surface area $\left(2630 \mathrm{~m}^{2} / \mathrm{g}\right)$, good resistance to oxidative processes, and high temperature stability [7]. Therefore, graphene has been considered an ideal electrode material for supercapacitors [7-15].

As a matter of fact, a flood of papers have demonstrated the promise of graphene in supercapacitor applications. They cover almost all aspects of the performance boost of supercapacitors. As discussed above, supercapacitors store energy mainly relying on two kinds of mechanisms. One is the electrochemical double-layer capacitance (EDLC), which results from charges absorbed in the interfaces between the electrodes and the electrolytes, i.e., the double layers. A high EDLC requires electrodes with high electrical conductivity and large surface area. In theory, supercapacitors based on graphene electrodes can achieve EDLC as high as $\sim 550 \mathrm{~F} / \mathrm{g}$ [16]. The other is the pseudo-capacitance, which is produced by highly reversible redox (faradic) reactions in electrode surfaces. Pseudo-capacitive materials typically include conducting polymers and a variety of transition metal oxides (e.g., $\mathrm{RuO}_{2}, \mathrm{MnO}_{2}$ and $\mathrm{IrO}_{2}$ ). In general, pseudo-capacitive materials can achieve higher capacitance than EDLC, despite their poor cycling stability [2,17]. Although graphene itself is not pseudo-capacitive, composites incorporating graphene with pseudo-capacitive materials represent an important possibility to fabricate high-performance and low-cost supercapacitors [11].

Recently, more attention has been paid to supercapacitors with asymmetric electrodes $[1,2,10,18]$, one of which is a capacitor-like electrode (as the power source), whereas the other is a battery-like faradaic electrode (as the energy source). An appropriate combination of electrodes in asymmetric supercapacitors can offer the advantages of both supercapacitors (charging rate and cycle life) and batteries (energy density). In particular, asymmetric supercapacitors may have evidently increased operation voltage that further improves the energy and power densities. Advanced asymmetric supercapacitors based on graphene electrodes have also been demonstrated to be of high energy density $[18,19]$.

Consequently, the high electrical conductivity, large specific surface area, great incorporation with various pseudo-capacitive materials, and suitability for asymmetric supercapacitors make graphene a highly suitable material for supercapacitors. These points have been well demonstrated in many other review papers [8-15]. However, in practice, people still have not been able to utilize the full potential of graphene for supercapacitors. The specific EDLC (typically around 100-200 F/g) for practical 
graphene-based supercapacitors falls far below the theoretical value [16]. An important technological challenge is the restacking of graphene flakes when they are packed into electrode structures, which strongly reduces the active surface area of graphene. The restacking is likely caused by strong sheet-to-sheet van der Waals interactions [16,20]. In recent studies, a large number of novel techniques have been developed to prevent such restacking for further performance improvement. In this review, we do not attempt to include all the studies on graphene-based supercapacitors, but only focus on those techniques which aim to prevent the restacking of graphene flakes and hence make the best use of, or even further increase, their large specific surface area.

\section{Advanced Techniques for Prevention of Graphene Restacking}

In this section, we systematically and briefly introduce recent advanced techniques (or strategies) which aim to prevent the restacking of graphene flakes to improve the electrochemical performance of the supercapacitors. These techniques are classified into several categories. For each category, we only introduce the most representative techniques.

\subsection{Vertically Oriented Graphene Sheets}

A near-ideal strategy to prevent graphene stacking is to make the graphene flakes vertically oriented, or perpendicular to the current collectors. In this way, the graphene surface area can be efficiently utilized. There have been at least two techniques which have realized this strategy: Growth of vertically oriented graphene nanosheets using chemical vapor deposition (CVD) [21] and "in-plane" ultrathin supercapacitors [22].

\subsubsection{CVD-Grown Vertical Graphene Nanosheets}

Miller et al. [21] used radio frequency plasma-enhanced CVD to synthesize vertically oriented graphene nanosheets directly on heated nickel (Ni) substrates, as shown in Figure 1.

Figure 1. (a) Scanning electron micrograph (SEM) image of vertically oriented graphene nanosheets grown on a Ni electrode; (b) SEM image of vertically oriented graphene nanosheets grown on a circular fiber. Reprinted with permission from [21]. Copyright 2010 American Association for the Advancement of Science.
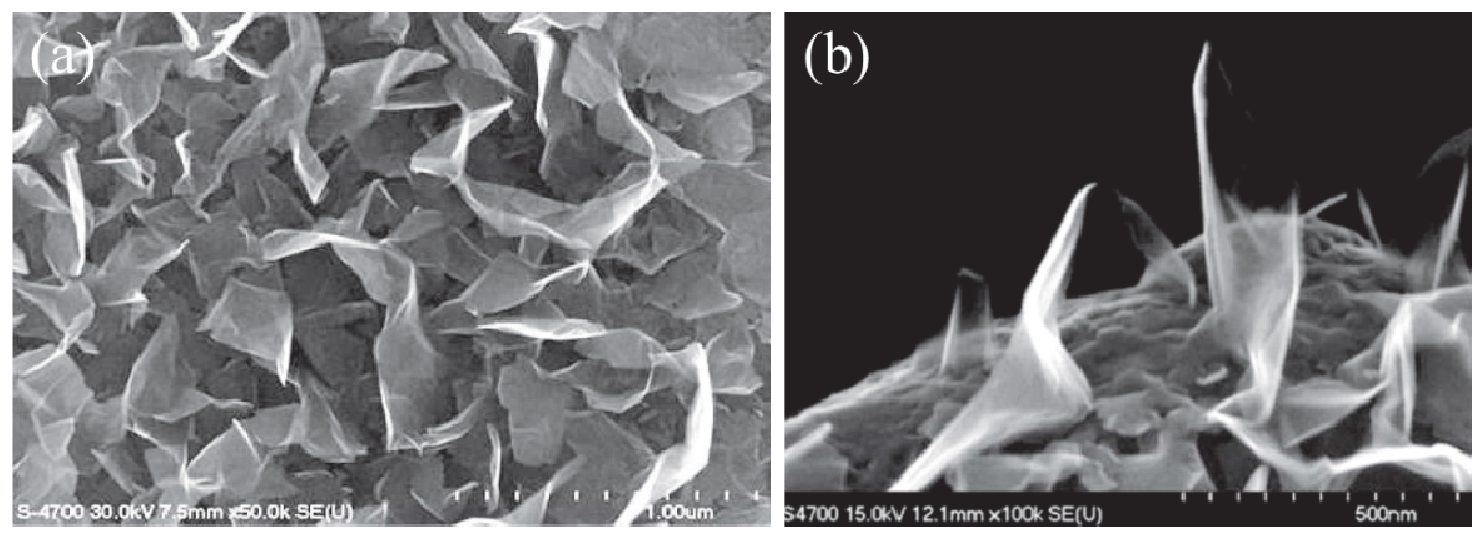
The process began with graphitic islands on the Ni substrate, and then the growth continued to form a basal layer which impinged the islands, so that the subsequent growth produced thin films composed of vertically oriented graphene nanosheets. As a result, most edge planes are exposed and directly accessible, giving rise to a large specific surface area of $\sim 1100 \mathrm{~m}^{2} / \mathrm{g}$ and hence providing a high capacitance density of $3 \mathrm{~F} / \mathrm{cm}^{3}$ with the thickness of only $0.6 \mu \mathrm{m}$. In addition, the graphene nanosheets are grown directly on the conductive substrate surface, which minimizes the series resistances and yields a resistor-capacitor time constant of less than $200 \mu \mathrm{s}$, which can efficiently filter $120 \mathrm{~Hz}$ current.

\subsection{2. "In-Plane" Graphene Supercapacitors}

As shown in Figure 2, the concept of "in-plane" graphene supercapacitors [22] provides another solution to vertically oriented graphene flakes. The key of this technique is that two separate graphene (or reduced graphene oxide, rGO) films are first fabricated with almost all graphene flakes lying horizontally on an electrically insulating substrate (see Figure 2c). Two metal current collectors are then deposited onto the substrate to connect the graphene electrodes, respectively. With this structure, almost all graphene flakes are perpendicular to the current collectors (see Figure 2b). The horizontal graphene flakes can be fabricated either through CVD growth on copper foils followed by a transfer to an insulating substrate, or through layer-by-layer self-assembly of solution-dispersed graphene flakes [22].

Figure 2. (a) Schematic illustration of the device fabricated using the concept of two dimensional (2D) "in-plane" supercapacitors; (b) Schematic illustration of the device structure (graphene flakes perpendicular to the current collectors) and operating principle for the "in-plane" supercapacitors; (c) SEM image of inner layers of the reduced graphene oxide (rGO) film indicating a flat-layered structure without any agglomeration. Adapted with permission from [22]. Copyright 2011 American Chemical Society.

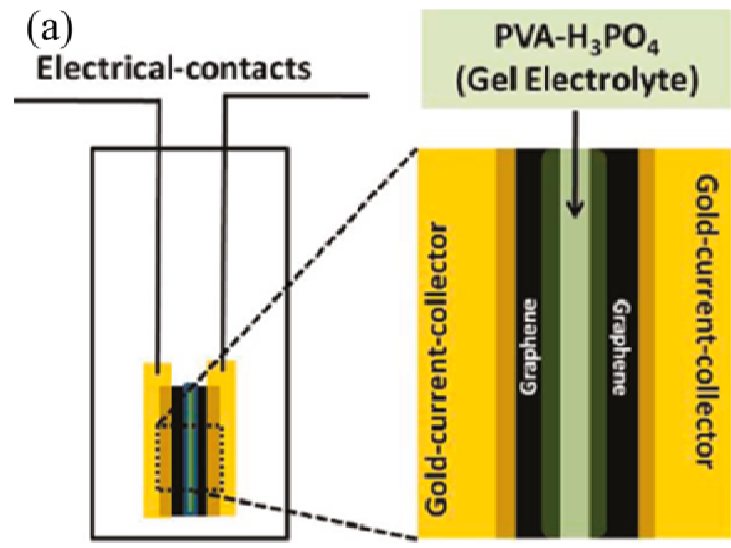

(b)

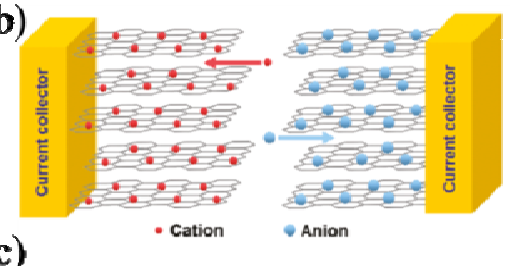

(c) - Cation Anion

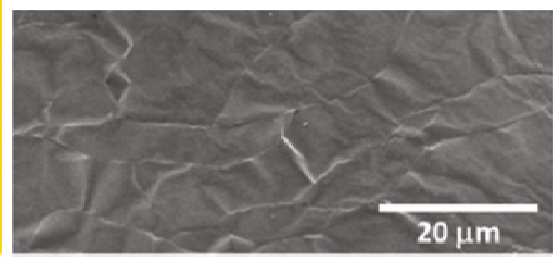

In addition to the advantages of the CVD-grown vertical graphene nanosheets (in Figure 1), the "in-plane" structure may offer opportunities to fabricate ultrathin supercapacitors, even including single-layer graphene supercapacitors. Ultrathin $(\sim 10 \mathrm{~nm}$ thick) graphene supercapacitors have achieved a high area-specific capacitance around $390 \mu \mathrm{F} / \mathrm{cm}^{2}$. 


\subsection{Deformed Graphene Sheets}

Recent studies have demonstrated that deformed graphene flakes, such as curved graphene [23], folded graphene [24], and crumpled graphene [25], can also increase the effective surface area to provide significantly improved performance for the supercapacitors.

\subsubsection{Curved Graphene Nanosheets}

Jang et al. [23] introduced a mesoporous graphene structure with a pore size ranging from 2 to $25 \mathrm{~nm}$. In particular, the mesoporous structure is accessible for ionic liquid electrolytes in spite of their large molecules and high viscosity. Supercapacitors based on such graphene electrodes can therefore achieve outstanding specific capacitance (up to $250 \mathrm{~F} / \mathrm{g}$ at a high current density of $1 \mathrm{~A} / \mathrm{g}$ ), high operating voltage $(4 \mathrm{~V})$ and an unprecedented specific energy of $\sim 86 \mathrm{Wh} / \mathrm{kg}$ at room temperature $\left(136 \mathrm{Wh} / \mathrm{kg}\right.$ at $\left.80^{\circ} \mathrm{C}\right)$.

The main feature of this mesoporous structure is that it is made of curved graphene nanosheets (see Figure 3a), rather than flat graphene flakes (Figure 3b). The curved morphology is likely capable of preventing graphene sheets from closely restacking with one another when they are packed or compressed into an electrode structure. In addition, the curved graphene nanosheets can be readily obtained by injecting graphene oxide (GO) suspension into a forced convection oven where a stream of compressed air is introduced to produce a fluidized-bed situation. Once the solvent or liquid is removed, the desired curved GO nanosheets are obtained which can be reduced by hydrazine to rGO nanosheets.

Figure 3. (a) SEM image of curved graphene nanosheets (scale bar $10 \mu \mathrm{m}$ ); (b) Transmission electron microscopy (TEM) image of flat graphene sheets prepared by a conventional chemical route (scale bar $500 \mathrm{~nm}$ ). Adapted with permission from [23]. Copyright 2010 American Chemical Society.
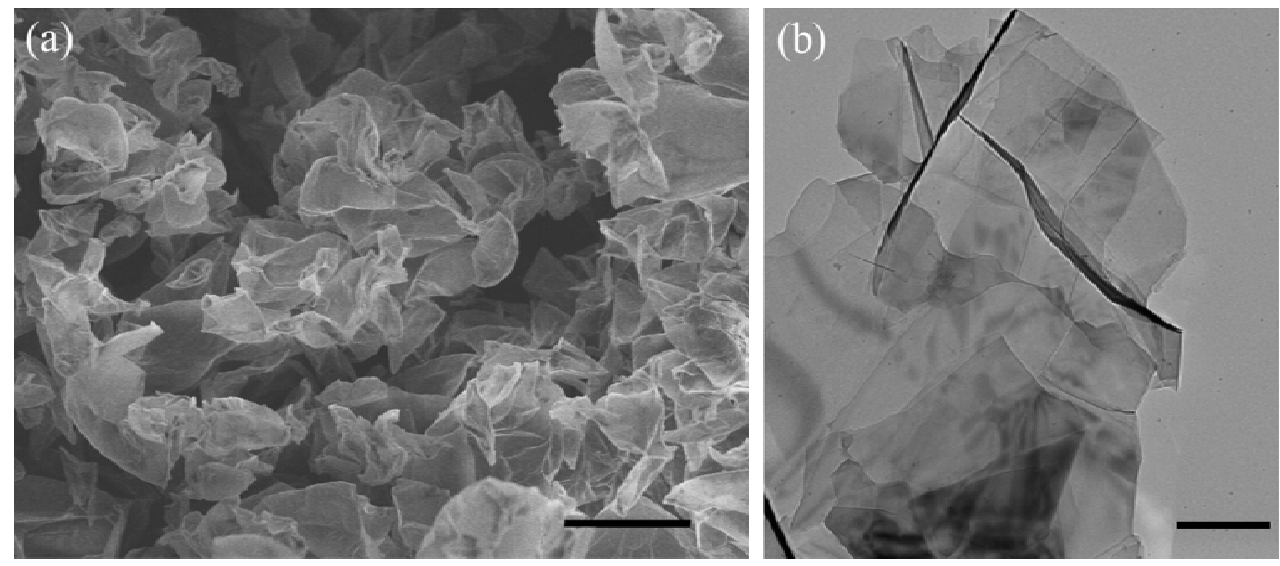

\subsubsection{Folded Graphene Nanosheets}

The folding of graphene structure (Figure 4) can also improve the electrochemical performance of supercapacitors. In contrast to the curved graphene, which effectively prevents restacking between different graphene sheets, folded graphene relies on its own folding which may provide slightly 
increased inter-sheet spacing and nucleation sites, as illustrated in Figure 4a. The folding can therefore enhance the accessibility of electrolyte and facilitate the formation of a solid-electrolyte interface leading to a higher reversible capacity [24].

The folded graphene can be fabricated by mechanically pressurizing a graphene aerogel, which is prefabricated by freeze-drying a GO aqueous dispersion and subsequent thermal reduction. Under mechanical pressure, the wrinkles and random curving of the sheets in the graphene aerogel undergo a fast deformation and transform into folds.

Figure 4. (a) Schematic illustration of folded graphene sheets; (b) and (c) SEM images of the folded graphene nanosheets. Adapted with permission from [24]. Copyright 2012 John Wiley and Sons.
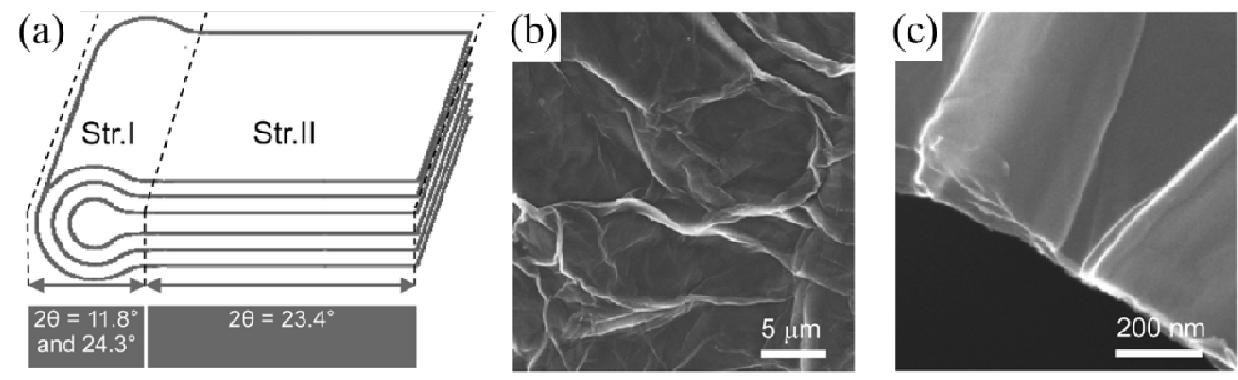

\subsubsection{Crumpled Graphene Nanosheets}

Chen et al. [25] developed a novel route to realize uniform nitrogen doping in graphene which gives rise to highly crumpled graphene nanosheets (Figure 5c,d). The crumpled nitrogen-doped graphene nanosheets provide a specific pore volume as high as $3.42 \mathrm{~cm}^{3} / \mathrm{g}$ (Figure $5 \mathrm{~d}$ ) while maintaining the 2D nanostructure of graphene with high conductivity, excellent mechanical strength and considerably large surface area. In addition, the nitrogen-rich graphene favors the long-term electrochemical stability and excellent cycling performance since the uniformly dispersed and covalently bonded molecular nitrogen groups are highly reversible and stable.

Figure 5. (a) Schematic illustration for fabrication of crumpled nitrogen-doped graphene sheets; (b) TEM image of pristine graphene oxide; (c) TEM and (d) SEM images of crumpled nitrogen-doped graphene sheets. Adapted with permission from [25]. Copyright 2012 John Wiley and Sons.

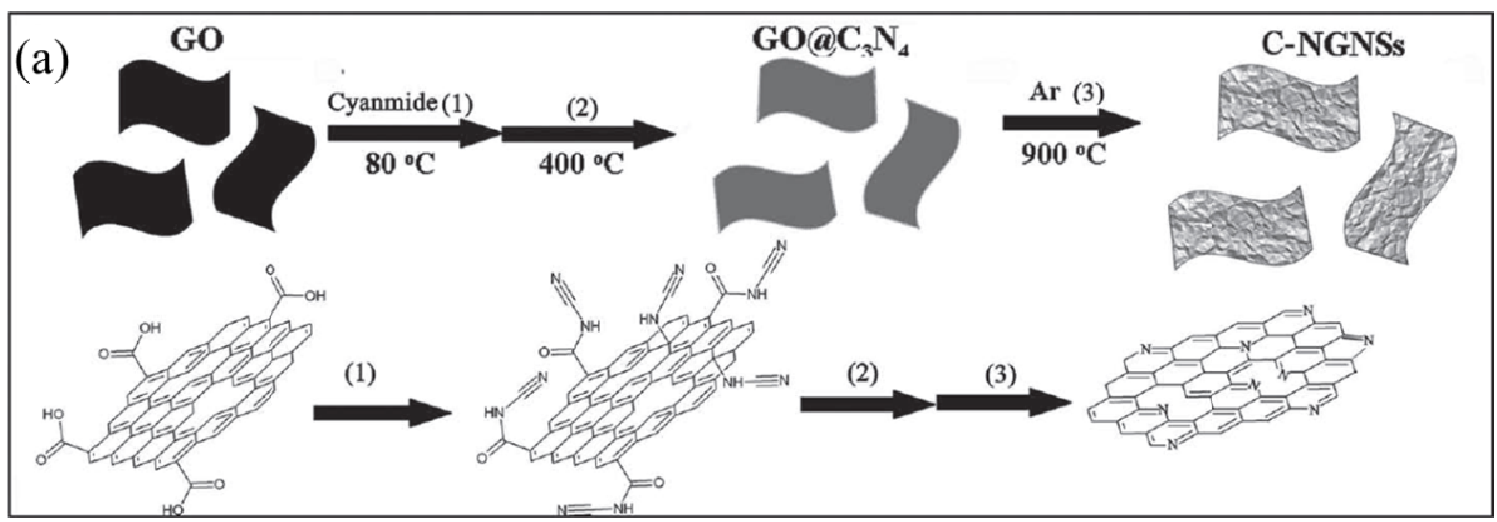


Figure 5. Cont.
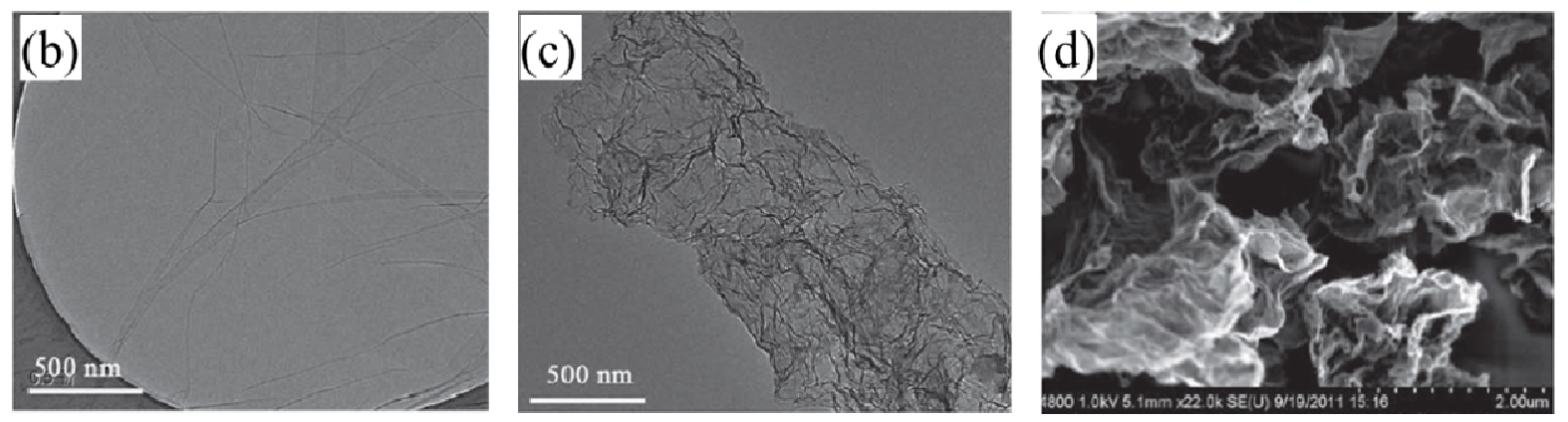

The synthesis of crumpled graphene nanosheets, as illustrated in Figure 5a, begins with mixing a GO solution with cyanamide $\left(\mathrm{NH}_{2} \mathrm{CN}\right)$ at an elevated temperature. During drying, some cyanamide reacts with the oxygen-containing groups in GO to form either amides or alkylammonium ions, while the rest is attached to the GO surface. The product is heated to $400{ }^{\circ} \mathrm{C}$ to polymerize the attached cyanamide and then calcined at $900{ }^{\circ} \mathrm{C}$ to reduce the $\mathrm{GO}$ and decompose the polymer simultaneously. The spices from the decomposed polymer provide nitrogen sources for finally evolving into the nitrogen-doped graphene, which contains many crumples (Figure 5c) as compared with the original GO (Figure 5b).

\subsection{Spaced Graphene}

Recent studies indicate that certain spacers (or stabilizers) can be intercalated into graphene layers to prevent the flake restacking. This is referred to as spaced graphene. Because of the existence of a variety of available spacer materials, such as nanoparticles, carbon nanotubes, and conducting polymers, spaced graphene represents the predominate techniques that attempt to make full use of graphene's large surface area. In terms of the type of spacer materials, the spaced graphene is categorized into different groups in this review. For each group, we mainly introduce the most representative and most recent examples.

\subsubsection{Metal Nanoparticles}

Si and Samulski [26] proposed a strategy of adhering metal (platinum) nanoparticles to the 2D graphene sheets to inhibit the aggregation (restacking) of graphene sheets, as shown in Figure 6. The metal nanoparticles increase the inter-sheet distance to several nanometers so that both faces of graphene sheets are accessible to the electrolyte. As a result, the induction of Pt nanoparticles increases the specific surface area and hence the specific capacitance of the graphene electrodes by about 20 times.

The Pt-graphene composite can be fabricated through chemical reduction of neutralized chloroplatinic acid $\left(\mathrm{H}_{2} \mathrm{PtCl}_{6}\right)$ using acid-functionalized graphene methanol solution. During the chemical reduction, it is essential to add a surfactant, 3-( $N, N$-dimethyldodecylammonio), to control the size of Pt nanoparticle and inhabit their aggregation (Figure 6d). This route to the Pt-graphene composite is anticipated to apply also to other metal nanoparticle-graphene composites [26]. 
Figure 6. (a) Schematic diagram of graphene nanosheets in dispersion and dry states; (b) Schematic diagram of nanoparticle-modified graphene nanosheets in dispersion and dry states; (c) SEM image of dried Pt-Graphene sheets; (d) TEM image of a thin slice of dried Pt-Graphene sheets. Adapted with permission from [26]. Copyright 2008 American Chemical Society.

(a)

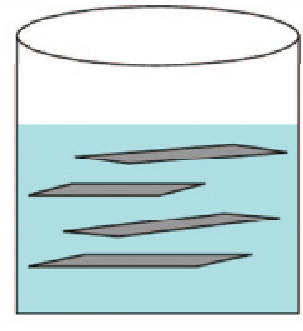

Dispersion of graphene

(b)

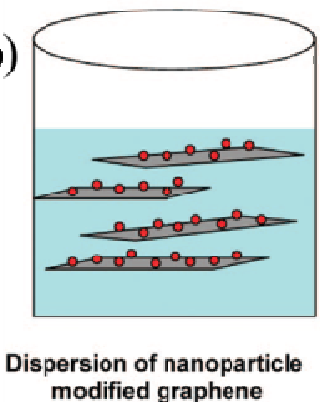

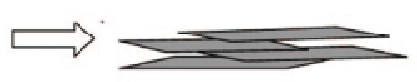

Graphene in dry state

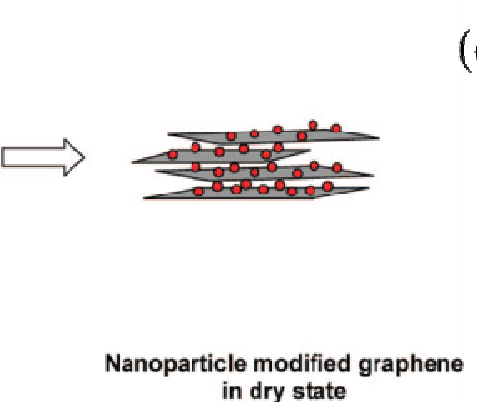

(c)

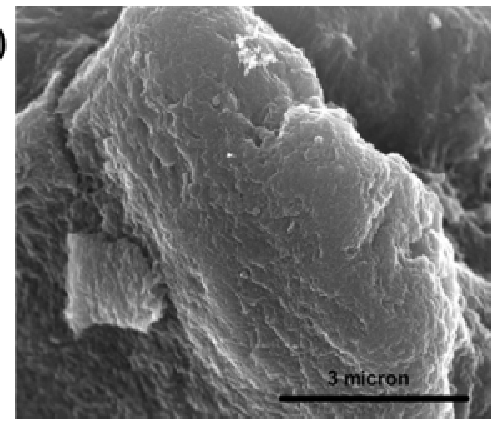

(d)

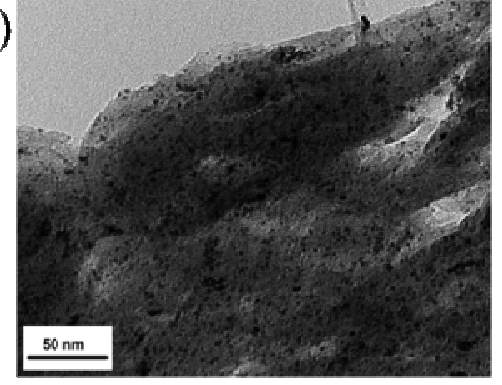

\subsubsection{Carbon Nanotubes}

Carbon nanotubes are an important allotrope of graphene. They are also promising for supercapacitor applications because of the high electrical conductivity and large specific surface area. Compared with graphene and many other carbon materials, the shortcomings of carbon nanotubes are their high production cost and low packing density. Nevertheless, carbon nanotubes are excellent spacers for graphene-based electrodes. In terms of the orientation of carbon nanotubes, there are horizontally aligned spacers and vertically aligned spacers.

The horizontally aligned carbon nanotube spacers, as illustrated in Figure 7a, can be realized in a straightforward manner. In principle, a simple procedure of mixing, drop casting and drying of graphene dispersion and carbon nanotube dispersion [27-29] can produce graphene films spaced by carbon nanotubes (Figure $7 \mathrm{~b}$ ), which is often called self-assembled graphene/carbon nanotube hybrid films. Usually, the inclusion of more than $10 \%$ (weight) carbon nanotubes can significantly improve the performance. In spite of the simplicity of this technique, a high specific capacitance of $>400 \mathrm{~F} / \mathrm{g}$ [27] and a high specific energy of $\sim 150 \mathrm{Wh} / \mathrm{kg}$ [28] can be achieved.

The vertically aligned carbon nanotube spacers usually rely on prefabricated three-dimensional (3D) graphene/carbon nanotube sandwich materials, where carbon nanotube pillars are grown in between the graphene layers [30], as shown in Figure 8. The 3D sandwich materials are fabricated by growing carbon nanotubes through CVD on the exfoliated GO with pre-deposited cobalt particles as the catalyst (Figure 8a). After thermal reduction, this unique sandwich structure can effectively utilize graphene's surface area and reduce the internal resistance. As a result, a high specific capacitance of $385 \mathrm{~F} / \mathrm{g}$ can be obtained. 
Figure 7. (a) Schematic diagram of graphene/carbon nanotube composite; (b) SEM image of a graphene/carbon nanotube composite. Adapted with permission from [29]. Copyright 2011 American Chemical Society.
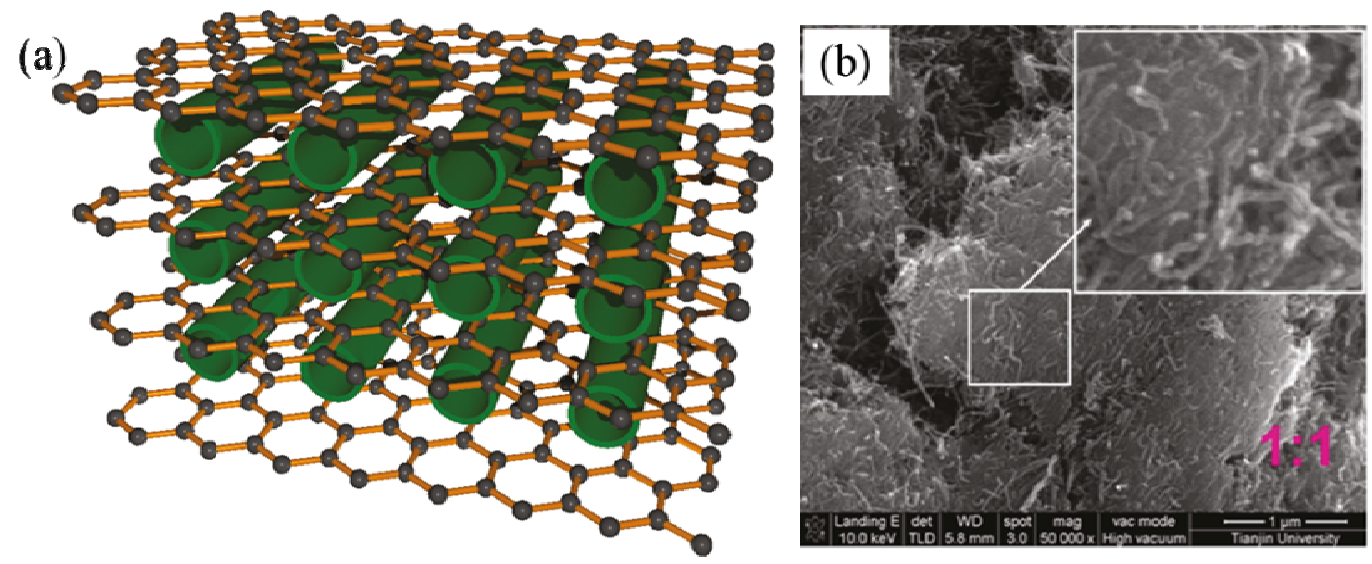

Figure 8. (a) Schematic illustration of the growth of the 3D sandwich materials with carbon nanotube pillars grown in between GO nanosheets; (b) and (c) SEM images of 3D graphene/carbon nanotube sandwich materials. Adapted with permission from [30]. Copyright 2010 John Wiley and Sons.

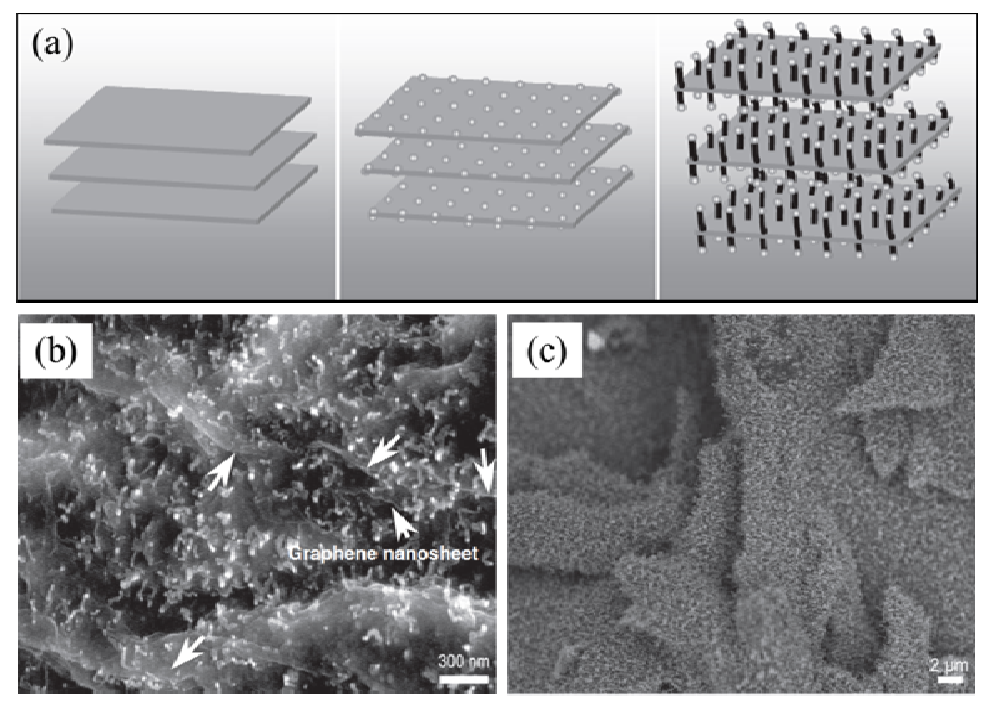

In particular, $\mathrm{Xu}$ et al. [31] synthesized 3D sponge-like graphene (Figure 9) which uses multi-walled carbon nanotubes (MWCNTs) as spacers. As shown in Figure 9a, the sponge-like graphene is synthesized by using MWCNTs and cobalt phthalocyanine (CoPc) molecules as precursors. Microwave heating and immediate quenching with ice water transform these materials into graphene flakes. During the reaction, $\mathrm{NH}_{3}$ and $\mathrm{HCl}$ gases are generated which act as dispersants to expand the materials to form sponge-like graphene. This sponge-like graphene can deliver a specific energy of $7.1 \mathrm{Wh} / \mathrm{kg}$ even at a high specific power of $48 \mathrm{~kW} / \mathrm{kg}$. In addition, they have excellent cycling stability with over $90 \%$ capacitance retention after 10,000 cycles. 
Figure 9. (a) Schematic illustration of the synthesis strategy for carbon nanotube-supported (sponge-like) graphene; (b) SEM and (c) TEM images of the 3D sponge-like graphene. Adapted with permission from [31]. Copyright 2012 American Chemical Society.

(a)
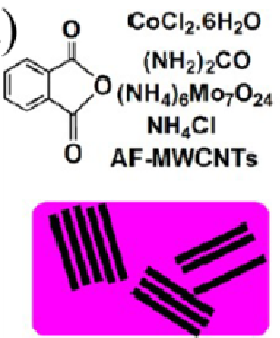

$$
\text { Dispersion }
$$
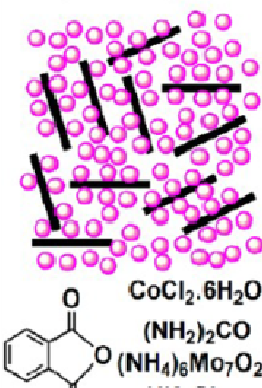

$\mathrm{NH}_{4} \mathrm{Cl}$

O AF-MWCNTs

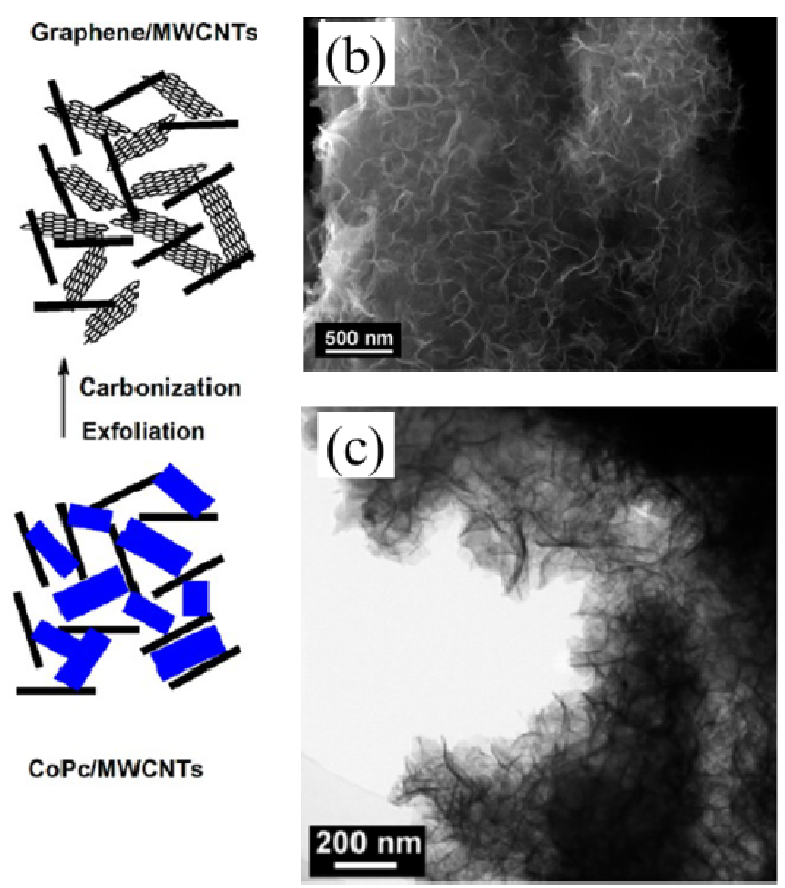

\subsubsection{Water (Solvated Graphene)}

It has recently been demonstrated [32] that water can also serve as an effective spacer to prevent the restacking of chemically converted graphene sheets (Figure 10). The solvated graphene films can be readily obtained from vacuum filtration of chemically converted graphene (or chemically reduced graphene oxide) dispersions followed by immediate transfer and immersion in water. Since freely suspended graphene sheets are usually corrugated, not perfectly flat, the corrugation may limit the inter-sheet contact area during graphene stacking. In addition, the carboxylic acid groups in the chemically converted graphene can cause electrostatic repulsions to prevent the stacking. More important, the hydrophilic groups can help to absorb water tightly onto the graphene surface so as to induce repulsive hydration forces between graphene sheets. The balance of these repulsive interactions and the inter-sheet $\pi-\pi$ attractions forms the special graphene/water hybrid structure. However, one should note that once dried, the solvated graphene structure is not recoverable because the dominant inter-sheet $\pi-\pi$ attractions can flatten the graphene sheets and make them difficult to be re-swollen in water. Nevertheless, the solvated graphene films can provide a specific capacitance of up to $215 \mathrm{~F} / \mathrm{g}$ (in an aqueous electrolyte) which is not sensitive to charge/discharge rates. In addition, the water in the graphene films can be easily exchanged by other solvents, such as ionic liquid. 
Figure 10. (a) and (b) Photographs of the as-formed flexible self-stacked solvated graphene films; (c) Schematic illustration of the cross-section of a solvated graphene film; (d) SEM image of the cross-section of a free-dried solvated graphene film; (e) XRD patterns of as-prepared and free-dried solvated graphene films. Reprinted with permission from [32]. Copyright 2011 John Wiley and Sons.
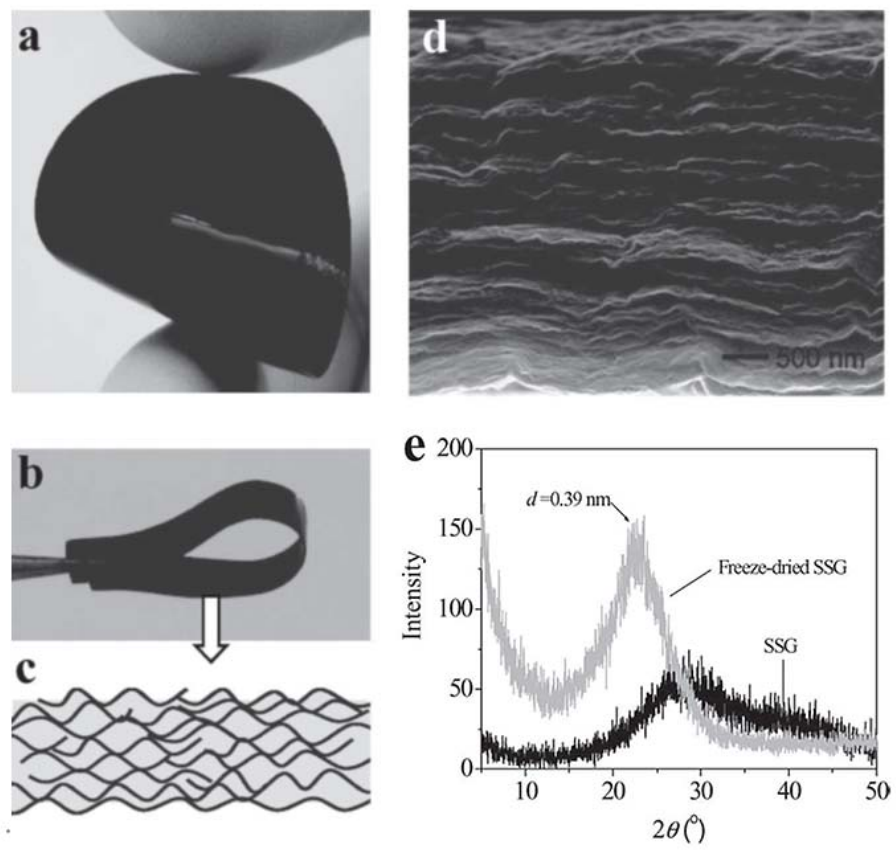

\subsubsection{Pseudo-Capacitive Metal Oxide Nanoparticles}

Pseudo-capacitive metal oxide nanoparticles are an important sort of spacers for graphene. Not only can they separate graphene nanosheets for more assessable surface area, but they also provide extra pseudo-capacitance to further improve the capacitance. A shortcoming of metal oxide is their poor conductivity. Usually, these metal oxides include $\mathrm{RuO}_{2}, \mathrm{MnO}_{2}, \mathrm{Ni}(\mathrm{OH})_{2}, \mathrm{NiO}, \mathrm{Fe}_{3} \mathrm{O}_{4}, \mathrm{CeO}_{2}, \mathrm{Co}_{3} \mathrm{O}_{4}$, $\mathrm{ZnO}, \mathrm{SnO}_{2}$, and so on $[10,15]$. Recent studies concerning the involvement of these metal oxides in more complicated graphene composites are introduced in this review, as discussed below.

\subsubsection{Conducting Polymers}

Conducting polymers, such as polyaniline (PANI), polypyrrole (PPy), and poly(3,4-ethylene-dioxythiophene):poly(styrenesulfonate) (PEDOT:PSS), have attracted great interest in studies on supercapacitors because of their very high pseudo-capacitance. Note that pseudo-capacitance relies on fast reversible redox reactions in the electrode surfaces, differing from EDLC, which arises from direct charge separation at the electrode/electrolyte interface. Conducting polymers often possess various oxidation states and the redox reactions involve transitions between different oxidation states. For example, redox reactions in PANI-based supercapacitors often involve two transitions: one is between a semiconducting state (leucoemeraldine) and a conducting state (polaronicemeraldine), and the other is between the emeraldine and pernigraniline forms $[33,34]$. Both of them can be identified by the peaks in cyclic voltammograms. A shortcoming of conducting polymers is their relatively poor cycling stability. Composites of conducting polymers with 
graphene [35] have been demonstrated as ideal electrode materials for supercapacitors because they can work synergistically to achieve both high energy density and improved cycling stability. Recently, advanced techniques [33-38] have also been developed to utilize conducting polymers as effective spacers for graphene electrodes to further increase the EDLC. The techniques include electrostatic interaction leading to PPy-sandwiched layered graphene [38], and grafting [33,34], direct coating [37], and vertically planting conducting polymers onto graphene [36].

A general problem for conducting polymer spacers is their weak interaction (van der Waals force) with graphene, which easily causes phase separation and hence degrades electrochemical performance. An effective way to enhance the graphene/polymer interaction is to graft conducting polymers into graphene sheets [33,34]. As illustrated in Figure 11, one strategy is that 4-aminophenol [33] is first introduced into GO sheets by covalent functionalization and subsequently polymerized in the presence of aniline monomer. Due to the thionyl chloride vapor used in the graft process, GO is reduced concomitantly, thereby yielding highly conducting networks. Another strategy [34] may involve a first graft of amide groups into GO sheets by covalent functionalization, followed by reaction of the GO derivative with PANI nanofibers and reduction of GO by hydrazine. The covalent functionalization ensures the compatibility of graphene with conducting polymer (PANI) and minimizes phase separation. As a result, a high specific capacitance over $600 \mathrm{~F} / \mathrm{g}$ has been attained [34].

Figure 11. Schematic illustration of the formation process of polyaniline-grafted graphene composites. Reprinted with permission from [33]. Copyright 2012 American Chemical Society.

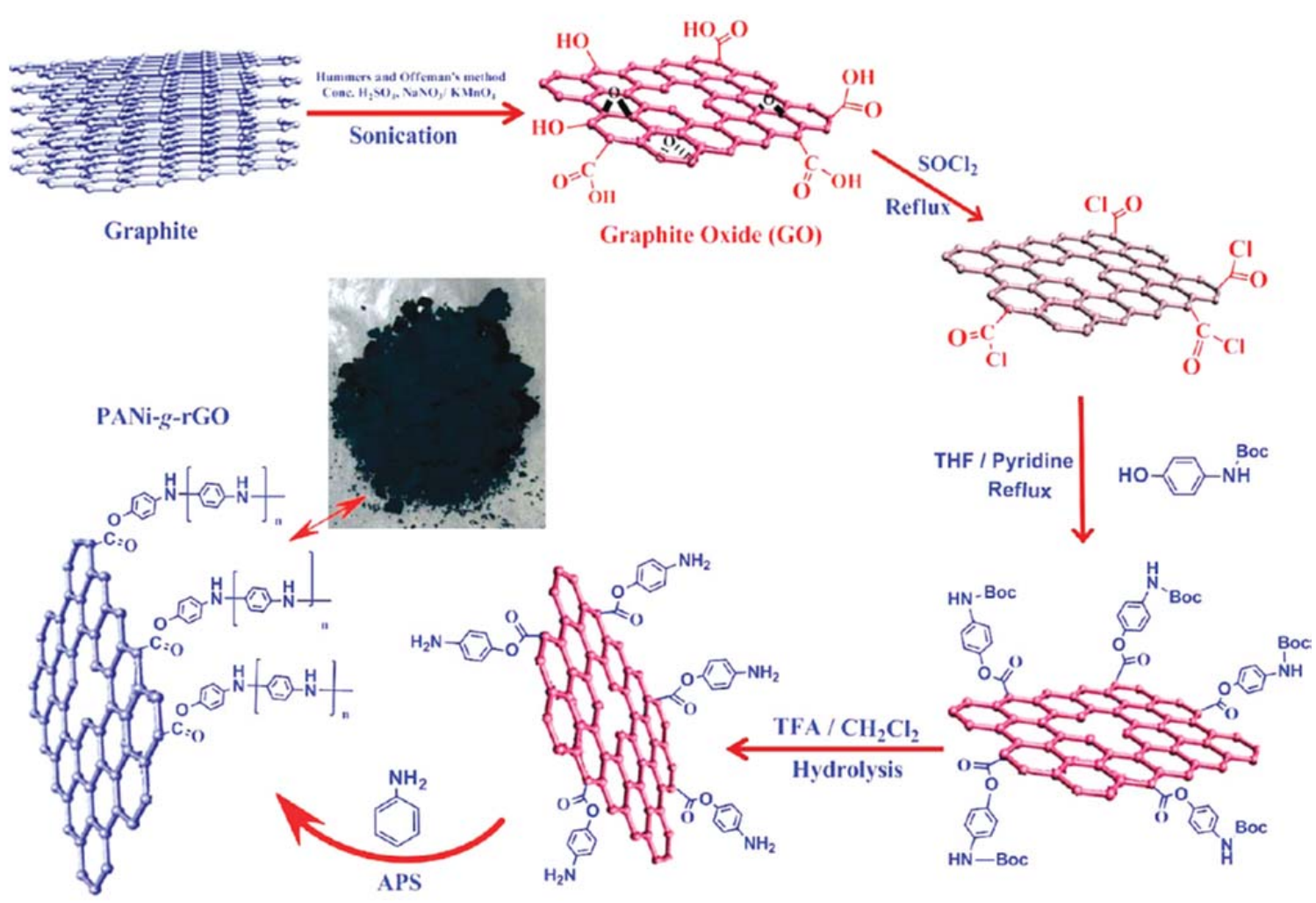


Zhang et al. [38] proposed another general route to fabricate layered graphene oxide with sandwiched conducting polymer. It is mainly based on electrostatic interactions between negatively charged GO sheets and positively charged surfactant micelles. Consequently, the surfactant micelles are adsorbed onto the graphene surface to form sandwiched structures (Figure 12). In addition, the hydrophobic cores of the surfactant micelles can attract conducting polymer monomer and after polymerization, layered GO structures pillared with the conducting polymers can be obtained. The demonstrated GO/PPy composite can provide a specific capacitance over $500 \mathrm{~F} / \mathrm{g}$.

Figure 12. Schematic illustration of the formation process of the sandwich-structured GO/PPy composites. Reprinted with permission from [38]. Copyright 2010 American Chemical Society.

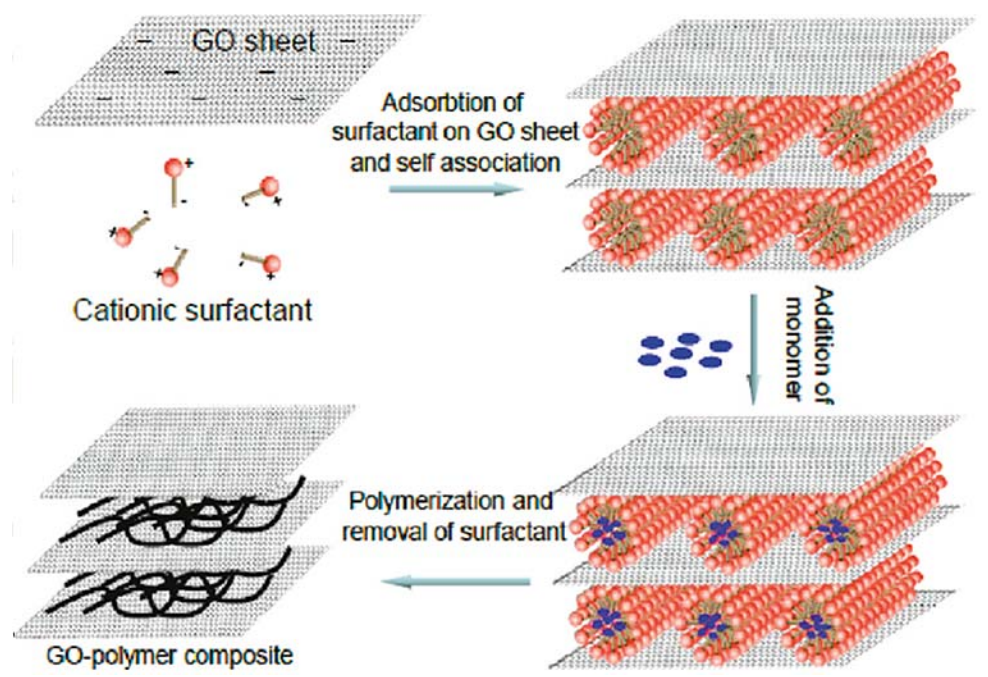

Like carbon nanotube, one attractive strategy for effective spacers is the vertical growth of conducting polymers onto graphene sheets. Xu et al. [36] introduced a facile method to fabricate such a composite (Figure 13). The aligned PANI nanowires were produced by dilute polymerization of aniline monomer in the GO aqueous solution. The morphologies of PANI nanowires were strongly affected by the aniline concentration and the polymerization temperature. Under an optimal concentration of $0.05 \mathrm{M}$ aniline and $0.36 \mathrm{mg} / \mathrm{mL} \mathrm{GO}$ in aqueous solution and with a low temperature of $-10{ }^{\circ} \mathrm{C}$, nucleation and vertical growth of PANI can uniformly take place on GO sheets. These composites of vertically oriented PANI on GO sheets can provide a high specific capacitance of $555 \mathrm{~F} / \mathrm{g}$ and good cycling stability.

Finally, further discussions are still necessary in order to fully understand the systems of graphene/conducting polymers and graphene/metal oxides. Although in this review we emphasize the function of conducting polymers and metal oxides as the spacers for graphene, they actually work synergistically, which means graphene actually also helps to disperse conducting polymers better or prevent metal oxides from agglomeration. 
Figure 13. SEM images of vertically grown polyaniline on graphene oxide sheets. Reprinted with permission from [36]. Copyright 2010 American Chemical Society.
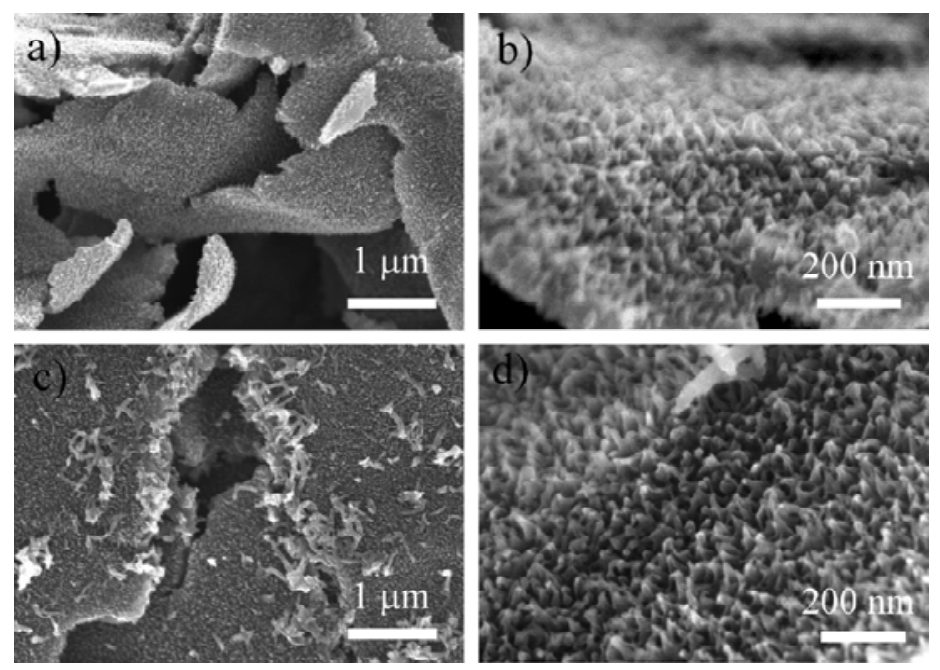

\subsubsection{Ternary Graphene Structure}

Although carbon nanotubes, metal oxides and conducting polymers are all excellent spacers for graphene electrodes, each of them has a significant shortcoming. Carbon nanotubes cannot provide pseudo-capacitance, metal oxides have low conductivity and conducting polymers are poor in cycling stability. Recent research tends to adopt the ternary structure which involves two kinds of the above spacers in addition to graphene so as to overcome the shortcoming and enhance the performance of the supercapacitors.

Rakhi et al. [39] demonstrated a ternary graphene- $\left(\gamma-\mathrm{MnO}_{2} /\right.$ carbon nanotube) structured nanocomposite (Figure 14). Firstly, $\gamma-\mathrm{MnO}_{2} /$ carbon nanotube composites were synthesized by dispersing functionalized carbon nanotubes in an aqueous solution containing $\mathrm{MnSO}_{4}$ through ultrasonication at an elevated temperature, followed by addition of $\mathrm{KMnO}_{4}$ under agitation. Then the graphene- $\left(\gamma-\mathrm{MnO}_{2} /\right.$ carbon nanotube $)$ composites were synthesized by ultrasonicating the mixture of chemically functionalized few-layered graphene nanosheets and the $\gamma-\mathrm{MnO}_{2} /$ carbon nanotube composites. The ternary graphene structure provides a high specific capacitance of $\sim 300 \mathrm{~F} / \mathrm{g}$ and excellent cycling stability (90\% retention after 5000 cycles).

Figure 14. (a) Schematic diagram of the ternary graphene- $\left(\gamma-\mathrm{MnO}_{2} /\right.$ carbon nanotube $)$ structure; (b) SEM image of the ternary graphene- $\left(\gamma-\mathrm{MnO}_{2} /\right.$ carbon nanotube) composite. Adapted with permission from [39]. Copyright 2012 John Wiley and Sons.
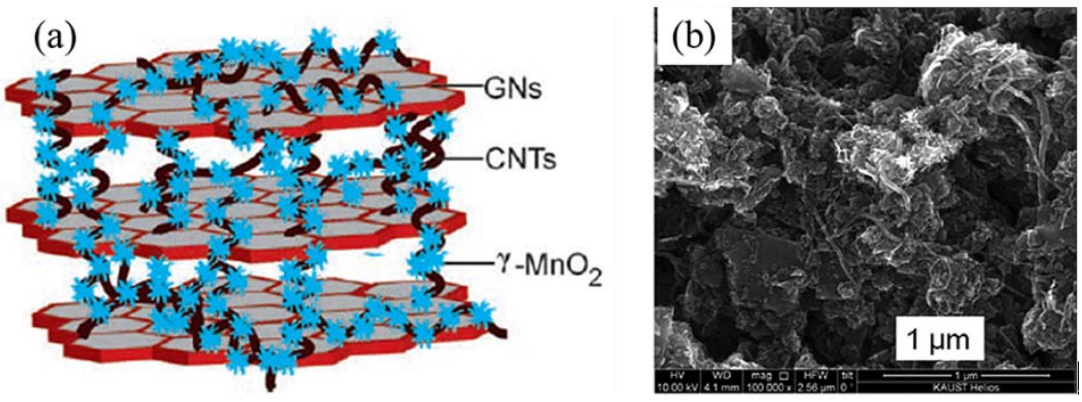
$\mathrm{Yu}$ et al. [40] demonstrated ternary (graphene $/ \mathrm{MnO}_{2}$ )-carbon nanotube and (graphene/ $\mathrm{MnO}_{2}$ )-conducting polymer structured composites (Figure 15). Firstly, the graphene/ $\mathrm{MnO}_{2}$ composites were synthesized through a two-step solution-based coating process [41]: Coating highly porous textile fibers with graphene nanosheets through a simple "dip and dry" process, followed by a controlled electrochemical deposition of $\mathrm{MnO}_{2}$ onto the as-prepared conductive textile fibers. Then, through again the "dip and dry" process, carbon nanotubes or conducting polymers (such as PEDOT:PSS) can be effectively coated to the graphene/ $\mathrm{MnO}_{2}$ textiles to form a $3 \mathrm{D}$ conductive wrapping. Such a ternary graphene structure provides a high specific capacitance of $\sim 380 \mathrm{~F} / \mathrm{g}$ with $>95 \%$ capacitance retention over 3000 cycles. Similarly, Cheng et al. [42] also fabricated such a ternary (graphene/ $\mathrm{MnO}_{2}$ )-carbon nanotube structured composite in a different way. The binary graphene $/ \mathrm{MnO}_{2}$ composite was firstly prepared using a sonication-assisted chemical co-precipitation method, and then mixed with few-walled carbon nanotube suspension under sonication to form the ternary composite. This composite provides a high specific capacitance of $372 \mathrm{~F} / \mathrm{g}$ with excellent rate capability in the absence of current collectors and binders.

Figure 15. (a) Schematic diagram of the ternary (graphene/ $\mathrm{MnO}_{2}$ )-carbon nanotube or (graphene/ $\mathrm{MnO}_{2}$ )-conducting polymer structure; (b) SEM image of the ternary (graphene/ $\mathrm{MnO}_{2}$ )-carbon nanotube composite; (c) SEM image of the ternary (graphene/ $\mathrm{MnO}_{2}$ )-PEDOT:PSS composite. Scare bar: $1 \mu \mathrm{m}$. Adapted with permission from [40]. Copyright 2011 American Chemical Society.
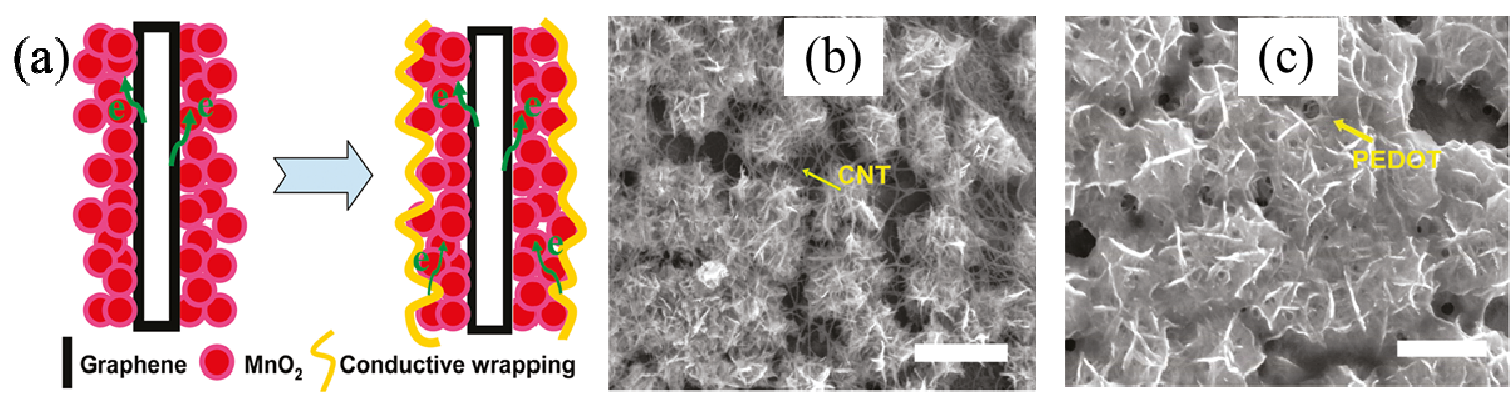

$\mathrm{Du}$ et al. [43] reported the fabrication of a ternary (graphene/carbon nanotube)-metal oxide composite (Figure 16). In particular, the binary (graphene/carbon nanotube) composite is of 3D-pillared architecture where carbon nanotubes are vertically grown on graphene (Figure 16a,b). To prepare the ternary composite, first the binary graphene/carbon nanotube was synthesized: Graphite was treated with acid and heated to become thermally expanded graphite, $\mathrm{SiCl}_{4}$ was introduced at high temperature through gas flow to form a $\mathrm{SiO}_{2}$ coating on the graphene layers, and then 3D-pillared graphene/carbon nanotube architecture was produced by pyrolysis of iron phthalocyanine. Finally, electrochemical deposition of $\mathrm{Ni}(\mathrm{OH})_{2}$ was employed to produce the $\mathrm{Ni}(\mathrm{OH})_{2}$-coated 3D-pillared graphene/carbon nanotube composite (Figure 16c,d). This 3D architecture provides a specific capacitance as high as $1065 \mathrm{~F} / \mathrm{g}$ even at a high current density of $22.1 \mathrm{~A} / \mathrm{g}$, with $96 \%$ capacitance retention after 20,000 cycles. 
Figure 16. (a) Schematic diagram of the 3D-pillared graphene/carbon nanotube structure; (b) SEM image of the 3D pillared graphene/carbon nanotube composite; (c) and (d) SEM images of the ternary $\mathrm{Ni}(\mathrm{OH})_{2}$-coated graphene/carbon nanotube composite. Adapted with permission from [43]. Copyright 2011 American Chemical Society.
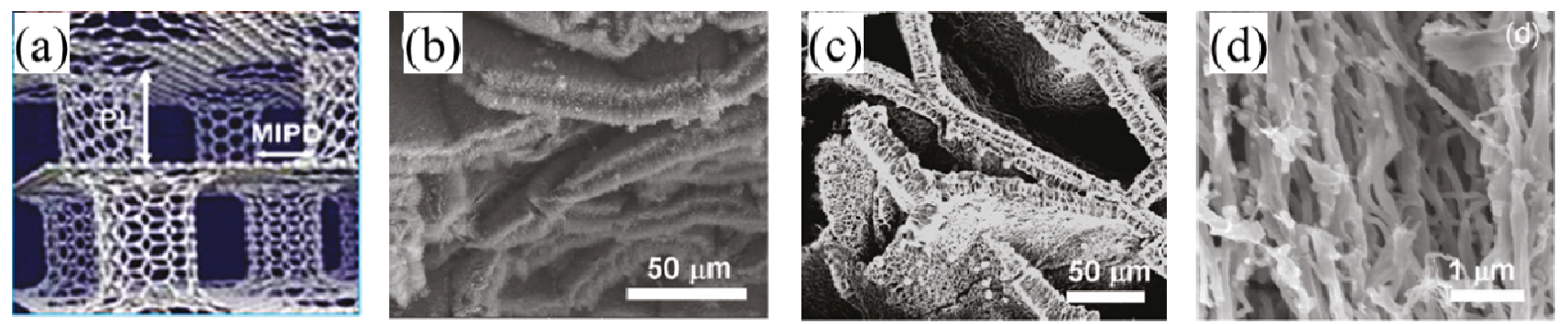

Yan et al. [44] demonstrated a ternary (graphene/PANI)-carbon nanotube structured composite (Figure 17), using a simple in situ polymerization method: Graphene (rGO) nanosheets and carbon nanotubes were dispersed in water through ultrasonication, and then aniline monomers were added and polymerized in the solution. In the ternary structure, graphene nanosheets act as support materials for deposition of PANI particles and carbon nanotubes as conductive wires interconnecting graphene/PANI particles (Figure 17a). This structure improves the composite conductivity and cycling stability because, during doping/de-doping processes, the highly conductive path, as well as mechanical strength of the electrode, can be maintained. As a result, the ternary composites achieve an extremely high specific capacitance of $1035 \mathrm{~F} / \mathrm{g}$ with $94 \%$ capacitance retention after 1000 cycles.

Figure 17. (a) Schematic illustration of the ternary (graphene/PANI)-carbon nanotube structure; (b) SEM image of the ternary composite (Inset is a corresponding TEM image). Adapted with permission from [44]. Copyright 2010 Elsevier.
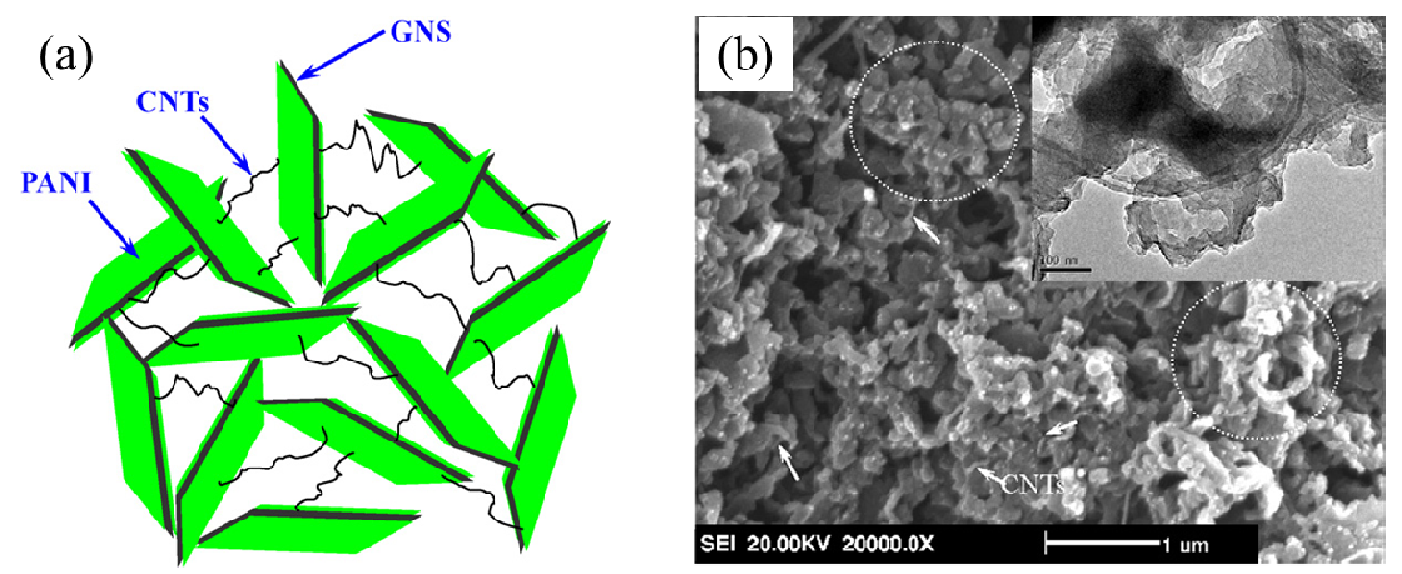

\subsection{D Graphene Networks}

A 3D graphene network refers to a 3D framework with assembled 2D graphene nano-sheets. In these structures, most graphene sheets stand on the supporting framework (usually cross-linked or etched porous structures/networks) so as to diminish the restacking problem and improve electrochemical performance. Several techniques have been introduced to fabricate 3D graphene structure for supercapacitor applications. 


\subsubsection{Graphene Hydrogels}

The simplest way to assemble 2D graphene nanosheets into complex 3D macrostructures might be the one-step hydrothermal reduction of graphene oxide aqueous dispersion to produce self-assembled graphene hydrogel [45]. The reduction process is typically through sealing GO aqueous dispersion in a Teflon-lined autoclave and maintaining at $180{ }^{\circ} \mathrm{C}$ for several hours. Initially, the GO sheets are randomly dispersed in water (Figure 18a,c). Upon reduction, the flexible graphene nanosheets begin to stack. If the graphene concentration is high enough, cross-linking may form through partial overlapping of graphene nanosheets (Figure 18c) and finally more and more cross-linking sites are generated yielding a 3D network with pore size ranging from sub-micrometer to micrometer (Figure 18b). Simultaneously, the residual oxygenated functional groups on the graphene sheets can entrap ample water into the graphene network to form a self-assembled graphene hydrogel. The hydrogel can provide a specific capacitance above $150 \mathrm{~F} / \mathrm{g}$.

Figure 18. (a) Photographs of a $2 \mathrm{mg} / \mathrm{mL}$ GO aqueous dispersion before and after hydrothermal reduction; (b) SEM image of the self-assembled graphene hydrogel; (c) Schematic illustration of the proposed formation mechanism for graphene hydrogel. Adapted with permission from [45]. Copyright 2010 American Chemical Society.
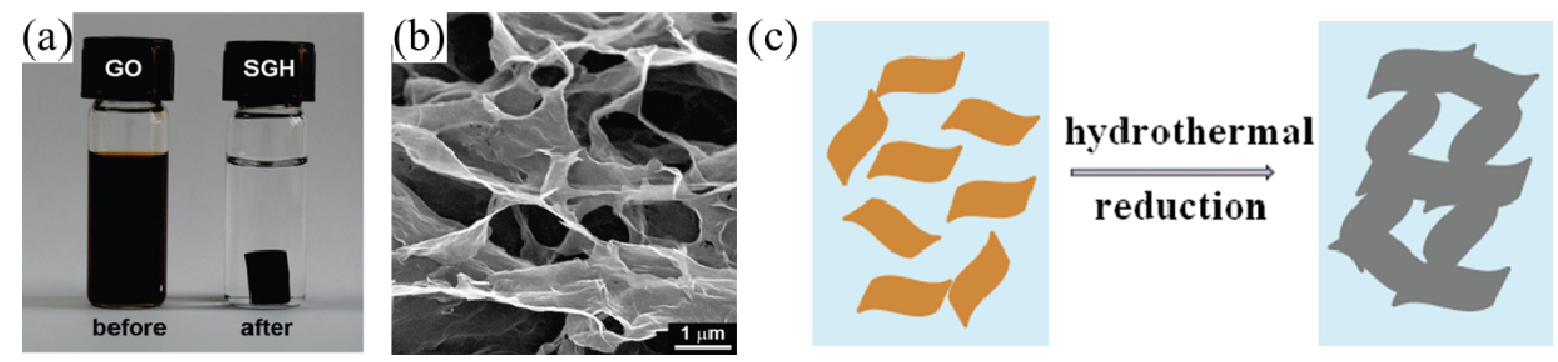

\subsubsection{Graphene Foams}

Graphene foams represent another type of 3D graphene networks. Several techniques have recently been proposed to fabricate graphene foams.

Niu et al. [46] introduced a leavening strategy to prepare graphene foams, as illustrated in Figure 19a. Leavening is the process of adding gas before or during baking or steaming in order to produce lighter, more easily chewable bread with a porous structure from the more compact dough [46]. In this technique, graphene oxide films are first prepared through vacuum filtration of GO solutions followed by peeling off. The free-standing GO films are put into hydrazine monohydrate in a sealed vessel and heated. The hydrazine vapor initiates the chemical reduction of GO to $\mathrm{rGO}$ with the rapid evolution of gaseous species which result in porous graphene foams. As shown in Figure 19b, the pore walls in the foams are continuously cross-linked, which effectively prevents the restacking of graphene sheets but also improves the inter-sheet contacts. The specific capacitance of these graphene foams is about $110 \mathrm{~F} / \mathrm{g}$. 
Figure 19. (a) Schematic illustration of the leavening process to prepare reduced graphene oxide foams; (b) SEM image of the graphene foam. Adapted with permission from [46]. Copyright 2012 John Wiley and Sons.
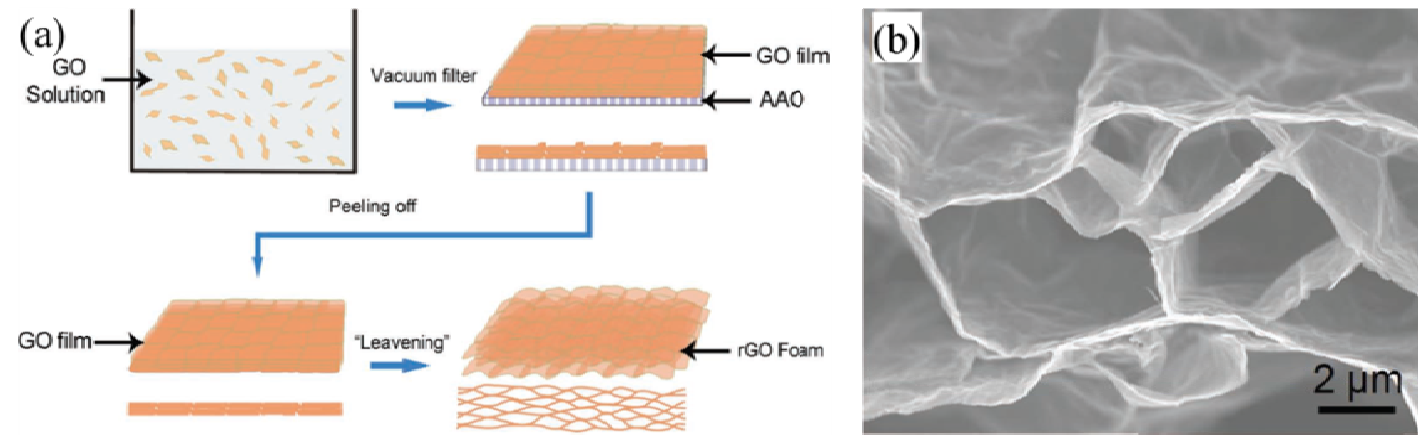

Cao et al. [47] introduced a template-assisted method to grow graphene foams. This technique uses $\mathrm{Ni}$ foam (Figure 20a) as the sacrificial template. Graphene is grown onto the template (Figure 20b) by CVD with ethanol as the carbon source. After removal of Ni foam through etching in $\mathrm{FeCl}_{3}$ and $\mathrm{HCl}$ solutions, the remaining graphene sheets replicate the $3 \mathrm{D}$ network and porous structure of Ni foam (Figure 20c). In addition, for performance enhancement, a metal oxide, such as NiO, can be coated onto the graphene foam through electrochemical deposition (Figure 20d). The obtained $\mathrm{NiO} /$ graphene composite exhibits a high specific capacitance of about $816 \mathrm{~F} / \mathrm{g}$ and a stable cycling performance without any capacitance loss after 2000 cycles.

Figure 20. (a) Photograph of Ni foam before and after the growth of graphene; (b) SEM image of 3D graphene network grown on Ni foam after CVD; (c) SEM image of 3D graphene network after removal of Ni foam; (d) SEM image of graphene foam coated with NiO. Adapted with permission from [47]. Copyright 2011 John Wiley and Sons.
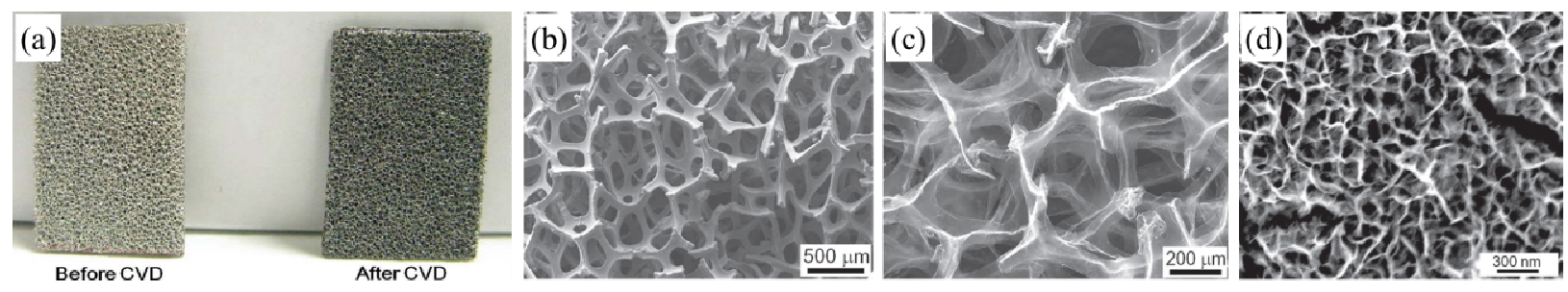

Zhao et al. [48] developed a strategy to fabricate PPy-mediated graphene foams (Figure 21a). The foams were prepared by hydrothermal reduction of GO aqueous suspension containing pyrrole monomers, followed by electrochemical polymerization of the pyrrole monomer in $\mathrm{NaClO}_{4}$ aqueous solution. The key step to PPy-graphene foams is the introduction of pyrrole monomer into the GO suspension. The conjugated structure with electron-rich $\mathrm{N}$ atom in pyrrole monomer may easily attach on the surfaces and galleries of GO sheets through H-bonding or $\pi-\pi$ interaction. Thus, the existence of pyrrole will effectively prevent the self-stacking behavior of GO during hydrothermal process, and accordingly increase the amount of available GO sheets for forming large volume of 3D graphene with thin connection walls (Figure 21b). The specific capacitance of these PPy-graphene foams is about $350 \mathrm{~F} / \mathrm{g}$ at a constant current of $1.5 \mathrm{~A} / \mathrm{g}$. In particular, they have excellent compression tolerance. There is no evident capacitance loss under a 50\% applied strain, even after 1000 cycles. 
Figure 21. (a) Photographs of the as-prepared 3D PPy-graphene foam; (b) SEM image of the 3D PPy-graphene foam (scale bar: $1 \mu \mathrm{m}$ ). Adapted with permission from [48]. Copyright 2013 John Wiley and Sons.

(a)

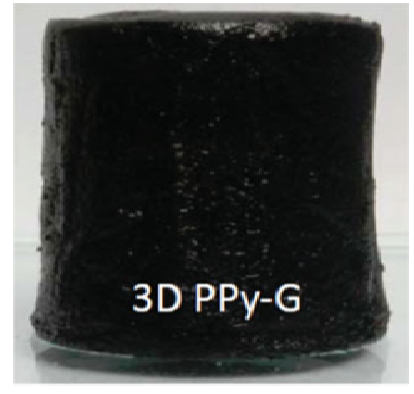

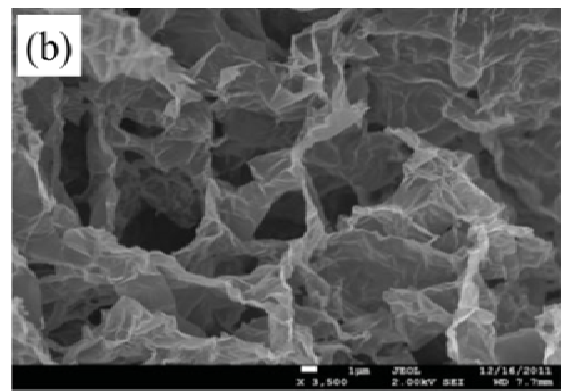

\subsubsection{D Nanographene Bulk Materials}

Biener et al. [49] proposed a top-down strategy to fabricate graphene-based bulk materials from low-cost polymer-derived carbon foams through the controlled removal of carbon atoms from a porous carbon network. The technique starts with a macroporous carbon network, highly cross-linked organic gel. After the gel is dried under ambient conditions, pyrolysis transforms the organic polymer into a porous $\mathrm{sp}^{2}$-bonded carbon network comprising both amorphous regions and multilayer graphene nanoplatelets. The graphene-based bulk materials are obtained from etching off the more reactive amorphous carbon components and a partial etching of multilayer graphite components by controlled burn-off in an oxidizing atmosphere at elevated temperatures (Figure 22). These materials achieve a specific capacitance of $105 \mathrm{~F} / \mathrm{g}$.

Figure 22. (a) Schematic of the polymer-based top-down approach; (b) Illustration of the mechanical robustness of a centimeter-sized nanographene bulk material sample; (c) Open macroporous network architecture composed of micrometer-sized pores and ligaments; (d) Internal structure of ligaments composed of curved and intertwined graphene sheets. Selected double-layer regions are marked by ovals, and a typical intensity profile across such a double-layer region is shown on the left. Reprinted with permission from [49]. Copyright 2012 John Wiley and Sons.

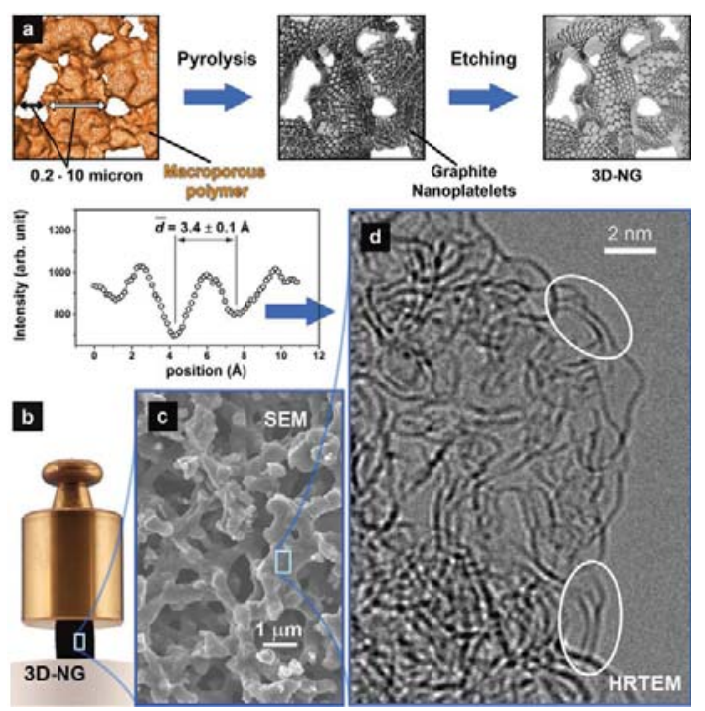




\subsubsection{Electrochemically Reduced Graphene Oxide}

Sheng et al. [50] developed a simple technique of electrochemically reducing graphene oxide to fabricate 3D graphene network. The electrochemically reduced graphene oxide electrode is prepared by electrolyzing $\mathrm{GO}$ aqueous suspension containing lithium perchlorate $\left(\mathrm{LiClO}_{4}\right)$ on a metal electrode (e.g., $\mathrm{Au}$ ) at an applied potential. This process reduces GO sheets and the resulting rGO sheets are self-assembled to form 3D interpenetrating networks (Figure 23) onto the substrate electrode. In the graphene networks, the pore walls are nearly vertical to the surface of current collectors (Figure 23b), so that they are fully exposed to the electrolyte for the access of ions to form electrochemical double-layers. These graphene electrodes exhibit both high area-specific capacitance $\left(\sim 0.5 \mathrm{mF} / \mathrm{cm}^{2}\right)$ and high rate (relaxation time constant $\sim 0.24 \mathrm{~ms}$ ).

Figure 23. SEM images of electrochemically reduced graphene oxide electrode. Adapted by permission from [50]. Copyright 2012 Macmillan Publishers Ltd.

(a)

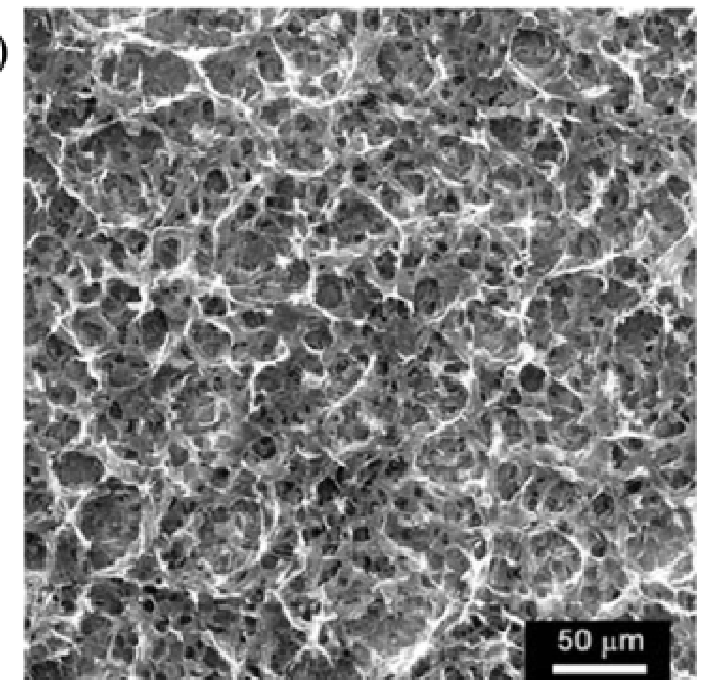

(b)

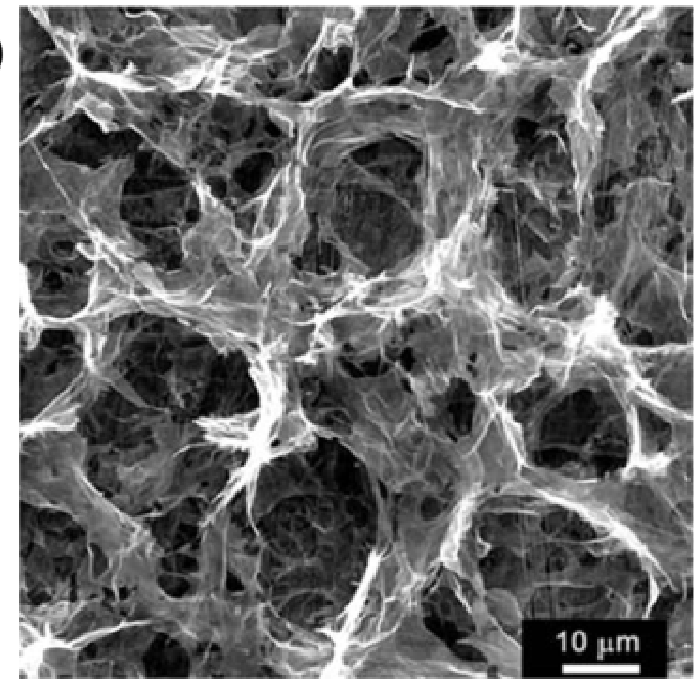

\subsubsection{Inkjet Printed Graphene Electrodes}

Recently, Li et al. [51] developed an inkjet printing technology to print micro-supercapacitors based on graphene electrodes. The graphene inks were prepared through a solvent exchange technique [52]. First, graphene is directly exfoliated from graphite in a solvent, and then the solvent is exchanged through distillation (Figure 24a) by another solvent which is compatible with inkjet printing. In order to extend the stable period, some polymers are added into the first solvent before distillation. After the inks were printed and dried, the graphene was embedded in the polymer matrix. Upon removal of the polymer by annealing, the remaining graphene formed a 3D network where most graphene flakes protrude from the surface (Figure 24b,c). The graphene electrodes exhibit an area-specific capacitance of $\sim 0.6 \mathrm{mF} / \mathrm{cm}^{2}$ and a fast resistor-capacitor time constant of $\sim 13 \mathrm{~ms}$. 
Figure 24. (a) Illustration of the distillation-assisted solvent exchange technique for preparation of graphene inks. Reprinted with permission from [52]. Copyright 2012 Elsevier; (b) and (c) SEM images of inkjet printed graphene electrodes for a micro-supercapacitor.

(a)

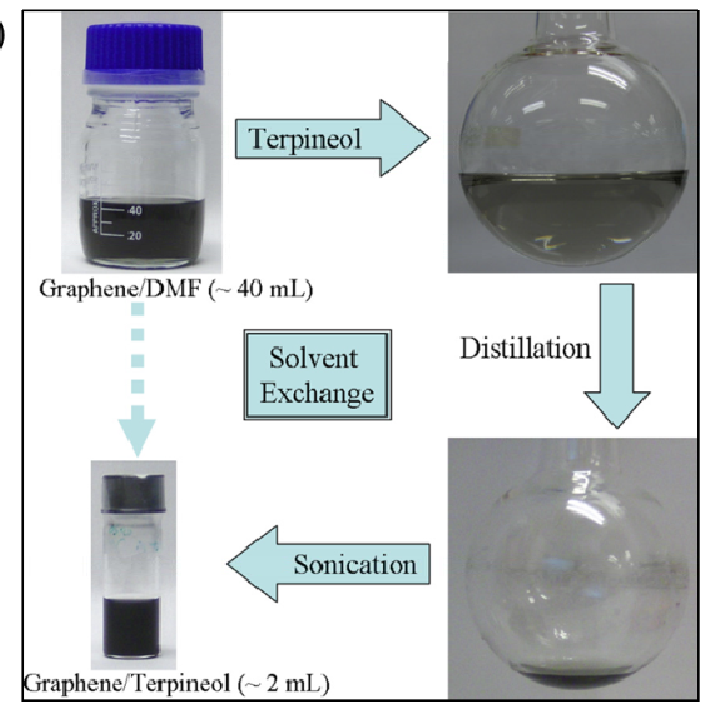

(b)

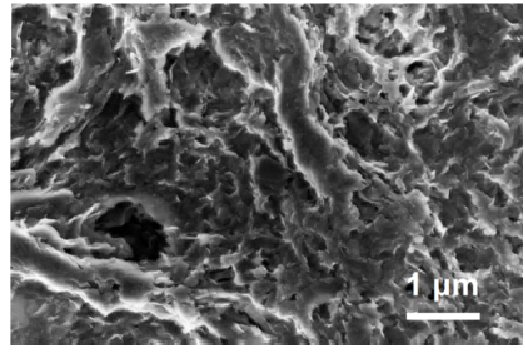

(c)

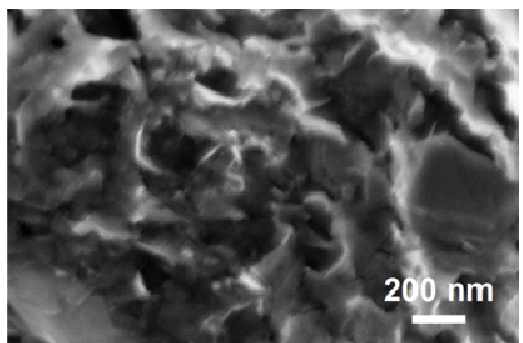

\subsubsection{Laser Scribed Graphene}

Laser scribing (Figure 25a) has been accepted as an effective method to reduce graphene oxide [16,53]. It is found [16] that the low-power infrared laser can change the stacked GO sheets (Figure 25b) into well-exfoliated few-layered rGO films. In particular, the laser produces open networks of rGO (Figure 25c), which prevents the restacking of graphene sheets. Supercapacitors based on the laser scribed graphene [16] provides high area-specific capacitance of $1.84 \mathrm{mF} / \mathrm{cm}^{2}$ even at a high current density of $1000 \mathrm{~A} / \mathrm{g}$, fast resistor-capacitor time constant of $33 \mathrm{~ms}$ and high stability of $96.5 \%$ capacitance retention after 10,000 cycles. In addition, micro-supercapacitors [53] based on laser scribed graphene may also have an area-specific capacitance of $0.51 \mathrm{mF} / \mathrm{cm}^{2}$.

Figure 25. (a) Schematic illustration of laser scribing to reduce graphene oxide; (b) SEM image of the stacked GO sheets before laser scribing; (c) SEM image of the porous graphene networks after laser scribing. Reprinted with permission from [16]. Copyright 2012 American Association for the Advancement of Science.
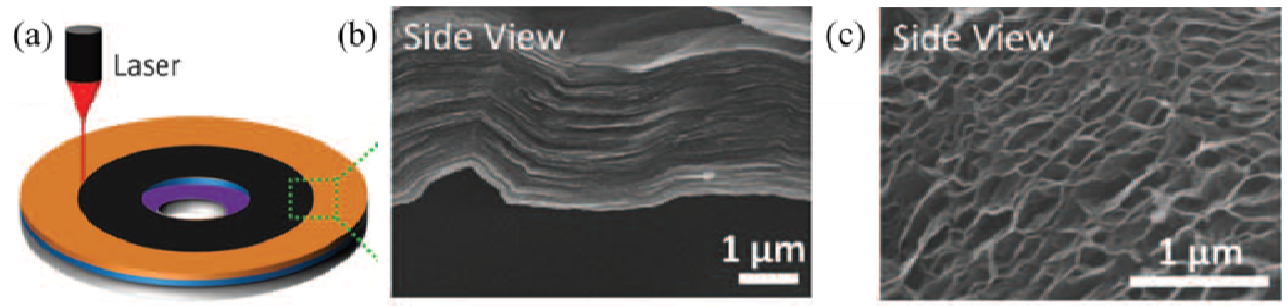

\subsubsection{Hierarchical Structure}

Recent studies tend to develop complex structures for 3D graphene networks by hierarchically combining more than one of the above techniques for further performance boost. 
Chen et al. [54] reported a simple strategy of depositing graphene hydrogel into the micropores of nickel foam to form a graphene hydrogel/nickel foam hierarchical composite electrode (Figure 26). Therefore, the graphene electrode and current collectors (nickel foams) are built in one piece, which decreases the device volume. Simultaneously, graphene hydrogel effectively forms electrochemical double layers and the nickel framework shortens the distances of charge transfer. As a result, supercapacitors with such electrodes (1 $\mathrm{mm}$ thick) exhibit high area-specific capacitance $\left(45.6 \mathrm{mF} / \mathrm{cm}^{2}\right)$, excellent cycling stability (stable after 10,000 cycles) and high rate capability (knee frequency about $38 \mathrm{~Hz}$ ).

Figure 26. (a) Photograph of a nickel foam; (b) Photograph of an as-prepared graphene hydrogel/nickel foam composite electrode; (c) SEM image of nickel foam; (d) SEM image of graphene hydrogel in the composite electrode. Adapted with permission from [54]. Copyright 2012 John Wiley and Sons.

(a)

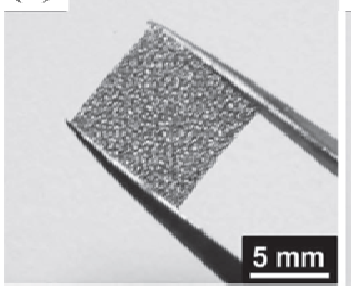

(b)

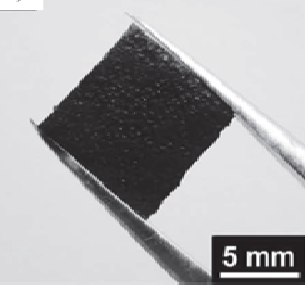

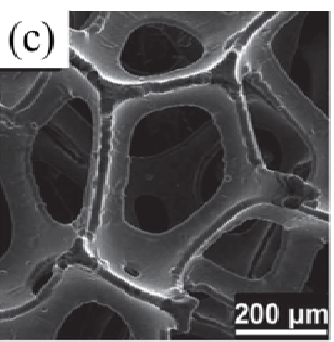

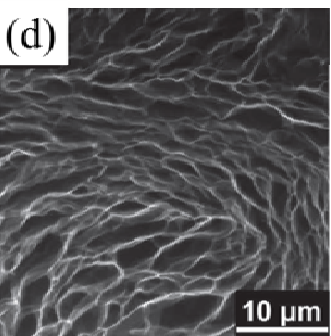

Dong et al. [55] demonstrated a hierarchical structure of graphene foam coated with $\mathrm{Co}_{3} \mathrm{O}_{4}$ nanowires. The graphene foam (Figure 27a) is fabricated through CVD with nickel foam as the substrate, and then $\mathrm{Co}_{3} \mathrm{O}_{4}$ nanowires are coated onto graphene foam through in situ hydrothermal synthesis. In this structure, graphene foam provides highly conductive pathways to ensure rapid charge transfer, and the $\mathrm{Co}_{3} \mathrm{O}_{4}$ nanowires effectively introduce pseudo-capacitance. Furthermore, the composite structure, graphene scaffold surrounded by $\mathrm{Co}_{3} \mathrm{O}_{4}$ nanowires (Figure 27b), can provide enormous accessible active area. Consequently, it can deliver a high specific capacitance of $\sim 1100 \mathrm{~F} / \mathrm{g}$ at a current density of $10 \mathrm{~A} / \mathrm{g}$ and be stable after $>500$ cycles.

Figure 27. (a) SEM image of the CVD-grown 3D graphene foam; (b) SEM image of $\mathrm{Co}_{3} \mathrm{O}_{4}$ nanowire-coated graphene foam. Reprinted with permission from [55]. Copyright 2012 American Chemical Society.

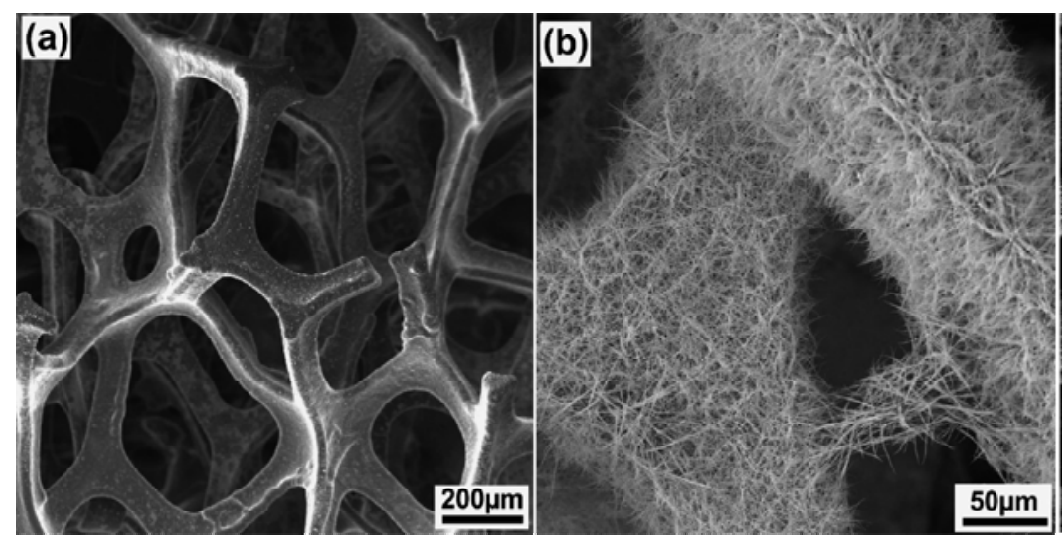




\section{Performance Overview}

Table 1 reviews the electrochemical performance for most of the supercapacitors fabricated from techniques included in Section 2. The listed values for all the parameters stand for the best performance reported for the devices. The electrolytes are usually aqueous solutions, unless otherwise specified. The cycling stability is characterized by the capacitance retention after a certain number of cycles. Note that for some devices, the capacitance may increase after cycling.

Table 1. Review of specific capacitance $\left(C_{\mathrm{s}}\right)$, specific power $\left(P_{\mathrm{s}}\right)$, specific energy $\left(E_{\mathrm{s}}\right)$, cycling stability, equivalent series resistance (ESR) and resistor-capacitor time constant $\left(t_{0}\right)$ for a variety of graphene-based supercapacitors. In several references, some parameters are not available, but instead their corresponding parameters are listed with explicitly indicated units.

\begin{tabular}{|c|c|c|c|c|c|c|c|}
\hline Graphene Type & $C_{\mathrm{s}}(\mathrm{F} / \mathrm{g})$ & $\begin{array}{c}P_{\mathrm{s}} \\
(\mathrm{kW} / \mathrm{kg})\end{array}$ & $\begin{array}{c}E_{\mathrm{s}} \\
(\mathrm{W} \mathrm{h} / \mathbf{k g})\end{array}$ & $\begin{array}{l}\text { Stability } \\
\text { (cycles/ } C_{\mathrm{s}} \\
\text { retention) }\end{array}$ & $\begin{array}{c}\text { ESR } \\
(\Omega)\end{array}$ & $\begin{array}{c}t_{0} \\
(\mathrm{~ms})\end{array}$ & References \\
\hline Vertical (CVD) & $3 \mathrm{~F} / \mathrm{cm}^{3}$ & - & - & - & 1.1 & 0.2 & {$[21]$} \\
\hline In-Plane & 247 & - & - & $1500 / \sim 100 \%$ & - & - & {$[22]$} \\
\hline Curved & $250^{\mathrm{a}}$ & $9.8^{\mathrm{a}}$ & $85.6^{\mathrm{a}}$ & - & 3.31 & - & {$[23]$} \\
\hline Folded & 172 & - & - & $>5000 />99 \%$ & - & - & {$[24]$} \\
\hline Crumpled & 302 & - & - & - & - & - & {$[25]$} \\
\hline Pt Spaced & 269 & - & - & - & - & - & {$[26]$} \\
\hline \multirow{3}{*}{$\begin{array}{c}\text { Horizontal Carbon } \\
\text { Nanotube (CNT) Spaced }\end{array}$} & 428 & 50 & 59.4 & $10,000 / 98 \%$ & $\sim 2$ & - & {$[27]$} \\
\hline & 290 & $58.5^{b}$ & $155.6^{\mathrm{a}}$ & $1000 / 129 \%{ }^{\mathrm{a}}$ & 1.68 & - & {$[28]$} \\
\hline & 318 & - & 11.1 & $1000 / 95 \%$ & - & - & [29] \\
\hline Vertical CNT Spaced & 385 & - & - & $2000 / 120 \%$ & - & - & {$[30]$} \\
\hline Sponge-Like & 80 & $48^{\mathrm{a}}$ & $21.4^{\mathrm{a}}$ & $10,000 / 98 \%$ & $\sim 7$ & 25 & {$[31]$} \\
\hline Solvated & $273^{a}$ & $776^{\mathrm{a}}$ & $150^{\mathrm{a}}$ & $100,000 / 97 \%$ & - & 13.3 & {$[32]$} \\
\hline \multirow{2}{*}{ PANI-Grafted } & 250 & - & - & - & - & - & {$[33]$} \\
\hline & 623 & - & - & $500 />100 \%$ & - & - & {$[34]$} \\
\hline PPy Sandwiched & 510 & - & - & $1000 / 70 \%$ & $<5$ & - & {$[38]$} \\
\hline Vertical PANI Coated & 555 & - & - & $2000 / 92 \%$ & - & - & {$[36]$} \\
\hline graphene- $\left(\gamma-\mathrm{MnO}_{2} / \mathrm{CNT}\right)$ & 308 & 26 & 43 & $5000 / 90 \%$ & 1.2 & - & [39] \\
\hline \multirow{2}{*}{$\left(\right.$ graphene/ $\left.\mathrm{MnO}_{2}\right)-\mathrm{CNT}$} & 320 & - & - & $3000 / 96 \%$ & 41 & - & {$[40]$} \\
\hline & 372 & 170 & 8.9 & $1000 / 95 \%$ & 2.4 & - & {$[42]$} \\
\hline $\begin{array}{c}\text { (graphene/ } \\
\mathrm{MnO}_{2} \text { )-PEDOT:PSS }\end{array}$ & 380 & - & - & $3000 / 95 \%$ & 27 & - & {$[40]$} \\
\hline $\begin{array}{l}\mathrm{Ni}(\mathrm{OH})_{2} \text {-pillared } \\
\text { graphene/CNT }\end{array}$ & 1384 & 8 & 35 & $20,000 / 96 \%$ & 3 & - & {$[43]$} \\
\hline (graphene/PANI)-CNT & 1035 & - & - & $1000 / 94 \%$ & 1.48 & - & [44] \\
\hline Hydrogel & 175 & - & - & - & - & - & {$[45]$} \\
\hline Foam (leavening) & 110 & - & - & - & - & - & {$[46]$} \\
\hline Foam (template)-NiO & 816 & - & - & $2000 / 120 \%$ & - & - & [47] \\
\hline Foam (PPy-mediated) & 350 & - & - & $1000 / \sim 100 \%$ & - & - & {$[48]$} \\
\hline Bulk (polymer-based) & 105 & - & - & - & - & - & [49] \\
\hline
\end{tabular}


Table 1. Cont.

\begin{tabular}{|c|c|c|c|c|c|c|c|}
\hline Graphene Type & $\begin{array}{c}C_{\mathrm{s}} \\
(\mathrm{F} / \mathrm{g})\end{array}$ & $\begin{array}{c}P_{\mathrm{s}} \\
(\mathrm{kW} / \mathrm{kg})\end{array}$ & $\begin{array}{c}E_{\mathrm{s}} \\
(W \mathrm{~h} / \mathrm{kg})\end{array}$ & $\begin{array}{l}\text { Stability } \\
\text { (cycles/ } C_{\mathrm{s}} \\
\text { retention) }\end{array}$ & $\begin{array}{c}\text { ESR } \\
(\boldsymbol{\Omega})\end{array}$ & $\begin{array}{c}\boldsymbol{t}_{0} \\
(\mathrm{~ms})\end{array}$ & References \\
\hline Electrochemical rGO & $0.9^{\mathrm{e}}$ & - & - & $10,000 / \sim 100 \%$ & 0.1 & 1.35 & {$[50]$} \\
\hline Inkjet Printed & $0.8^{\mathrm{e}}$ & $8.8 \mathrm{~mW} / \mathrm{cm}^{2}$ & $41 \mathrm{nW} \mathrm{h} / \mathrm{cm}^{2}$ & - & 8.7 & 12.5 & [51] \\
\hline \multirow{2}{*}{ Laser Scribed } & $276^{a}$ & $\sim 20^{\mathrm{a}, \mathrm{c}}$ & $1.36^{\mathrm{a}, \mathrm{d}}$ & $10,000 />97 \%$ & 16 & 33 & {$[16]$} \\
\hline & $0.5^{\mathrm{e}}$ & $\sim 15^{\mathrm{c}}$ & $4^{b, d}$ & $10,000 / 65 \%$ & 126 & - & [53] \\
\hline Hydrogel on Ni Foam & $46^{\mathrm{e}}$ & - & - & $2000 / 90 \%$ & 0.9 & 26 & [54] \\
\hline $\mathrm{Co}_{3} \mathrm{O}_{4}$-Graphene Foam & 1100 & - & - & $1000 />100 \%$ & 3 & - & {$[55]$} \\
\hline
\end{tabular}

From Table 1, it is clear that most techniques have significantly improved the specific capacitance, among which the ternary structures (spaced graphene with carbon nanotubes and pseudo-capacitive materials $[43,44]$ ) and hierarchical structure (graphene foam with metal oxide [55]) even have specific capacitance exceeding $1000 \mathrm{~F} / \mathrm{g}$. These devices also exhibit excellent cycling stability and relatively low ESR. However, one should note that both high specific power and high specific energy can be achieved even for low-specific-capacitance supercapacitors if they are compatible with ionic liquid as the electrolytes $[23,28,32]$. Therefore, it can be inferred that the combination between prevention of graphene restacking and introduction of pseudo-capacitance should represent a promising direction for design of supercapacitor electrodes, while the integration with ionic liquid as the electrolytes, which also benefits from the prevention of graphene restacking [23], is an important strategy to further improve the specific power/energy densities.

\section{Conclusions and Outlook}

We have reviewed recent research on preventing flake restacking for performance improvement in graphene-based supercapacitors. A variety of techniques and/or strategies have been demonstrated to make better use of the large specific surface area of graphene, ranging from proper placement of graphene (vertically oriented graphene), to modification of graphene (deformed graphene and spaced graphene), to construction of 3D graphene networks. These techniques have significantly improved the electrochemical performance of graphene-based supercapacitors. The performance boost can be clearly seen from the review in Table 1, where most techniques achieve a high specific capacitance over $200 \mathrm{~F} / \mathrm{g}$ (or equivalent). It further confirms that graphene is really a promising material for supercapacitor applications and that suppressing flake restacking provides an effective way to achieve higher performance for the supercapacitors.

Because of more and more demanding requirements from emerging applications, a further performance boost is still indispensable for graphene-based supercapacitors. Based on the attained performance among all the techniques included in this review, we predict several trends for future researches in this field: (1) The development of novel techniques to prevent graphene restacking is of continuous interest. A technique is especially favorable if it can bring a thorough improvement of all the parameters, rather than only one or a few of them, including specific capacitance, specific power, 
specific energy, cycling stability and frequency response, and so forth; (2) Incorporation of the existing techniques for preventing graphene restacking with other techniques/strategies may represent another important direction for future research. For example, many of the above techniques may be incorporated with ionic liquid electrolyte, and/or asymmetric-electrode architecture for further performance improvement; (3) Demonstration of new ternary structures for spaced graphene and hierarchical structures for 3D graphene network will continue to attract great interest since they are promising to synergistically take full advantage of the large surface area and excellent electrochemical stability of graphene, and the pseudo-capacitance of conducting polymers or metal oxides to simultaneously achieve high power/energy density, excellent cycling stability, and high rate. However, for such composite structures, the issues of co-existence and potential windows among different active materials should be carefully addressed; (4) More attention will be paid to research on application-oriented supercapacitors, such as current filters, compressible supercapacitors, flexible supercapacitors, and micro-supercapacitors. We hope this review can provide useful information and inspiration for future studies to expedite the development of graphene-based supercapacitors.

\section{Acknowledgments}

Supports from the European Research Council through the Advanced Investigator Grant OSIRIS (No. 228229) and VINN Excellence iPack Center, KTH are gratefully acknowledged.

\section{Conflict of Interest}

The authors declare no conflict of interest.

\section{References}

1. Simon, P.; Gogotsi, Y. Materials for electrochemical capacitors. Nat. Mater. 2008, 7, 845-854.

2. Miller, J.R.; Simon, P. Electrochemical capacitors for energy management. Science 2008, 321, $651-652$.

3. Conway, B.E. Electrochemical Supercapacitors: Scientific Fundamentals and Technological Applications; Kluwer Academic/Plenum: New York, NY, USA, 1999.

4. Chmiola, J.; Largeot, C.; Taberna, P.-L.; Simon, P.; Gogotsi, Y. Monolithic carbide-derived carbon films for micro-supercapacitors. Science 2010, 328, 480-483.

5. Geim, A.K.; Novoselov, K.S. The rise of graphene. Nat. Mater. 2007, 6, 183-191.

6. Chen, J.-H.; Jang, C.; Adam, S.; Fuhrer, M.S.; Williams, E.D.; Ishigami, M. Charged-impurity scattering in graphene. Nat. Phys. 2008, 4, 377-381.

7. Novoselov, K.S.; Fal'ko, V.I.; Colombo, L.; Gellert, P.R.; Schwab, M.G.; Kim, K. A roadmap for graphene. Nature 2012, 490, 192-200.

8. Zhu, Y.; Murali, S.; Cai, W.; Li, X.; Suk, J.W.; Potts, J.R.; Ruoff, R.S. Graphene and graphene oxide: synthesis, properties, and applications. Adv. Mater. 2010, 22, 3906-3924.

9. Luo, B.; Liu, S.; Zhi, L. Chemical approaches toward graphene-based nanomaterials and their applications in energy-related areas. Small 2012, 8, 630-646. 
10. Huang, Y.; Liang, J.; Chen, Y. An overview of the applications of graphene-based materials in supercapacitors. Small 2012, 8, 1805-1834.

11. Huang, X.; Zeng, Z.; Fan, Z.; Liu, J.; Zhang, H. Graphene-based electrodes. Adv. Mater. 2012, 24, 5976-6004.

12. Jiang, H.; Lee, P.S.; Li, C. 3D carbon based nanostructures for advanced supercapacitors. Energy Environ. Sci. 2013, 6, 41-53.

13. Brownson, D.A.C.; Kampouris, D.K.; Banks, C.E. An overview of graphene in energy production and storage applications. J. Power Sources 2011, 196, 4873-4885.

14. Grande, L.; Chundi, V.T.; Wei, D.; Bower, C.; Andrew, P.; Ryhänen, T. Graphene for energy harvesting/storage devices and printed electronics. Particuology 2012, 10, 1-8.

15. Choi, H.-J.; Jung, S.-M.; Seo, J.-M.; Chang, D.W.; Dai, L.; Baek, J.-B. Graphene for energy conversion and storage in fuel cells and supercapacitors. Nano Energy 2012, 1, 534-551.

16. El-Kady, M.F.; Strong, V.; Dubin, S.; Kaner, R.B. Laser scribing of high-performance and flexible graphene-based electrochemical capacitors. Science 2012, 335, 1326-1330.

17. Lai, L.; Yang, H.; Wang, L.; Teh, B.K.; Zhong, J.; Chou, H.; Chen, L.; Chen, W.; Shen, Z.; Ruoff, R.S.; Lin, J. Preparation of supercapacitor electrodes through selection of graphene surface functionalities. ACS Nano 2012, 6, 5941-5951.

18. Yan, J.; Fan, Z.; Sun, W.; Ning, G.; Wei, T.; Zhang, Q.; Zhang, R.; Zhi, L.; Wei, F. Advanced asymmetric supercapacitors based on $\mathrm{Ni}(\mathrm{OH})_{2} /$ graphene and porous graphene electrodes with high energy density. Adv. Funct. Mater. 2012, 22, 2632-2641.

19. Wu, Z.-S.; Ren, W.; Wang, D.-W.; Li, F.; Liu, B.; Cheng, H.-M. High-energy $\mathrm{MnO}_{2}$ nanowire/graphene and graphene asymmetric electrochemical capacitors. ACS Nano 2010, 4, 5835-5842.

20. Shen, J.; Hu, Y.; Li, C.; Qin, C.; Ye, M. Synthesis of amphiphilic graphene nanoplatelets. Small 2009, 5, 82-85.

21. Miller, J.R.; Outlaw, R.A.; Holloway, B.C. Graphene double-layer capacitor with ac line-filtering performance. Science 2010, 329, 1637-1639.

22. Yoo, J.J.; Balakrishnan, K.; Huang, J.; Meunier, V.; Sumpter, B.G.; Srivastava, A.; Conway, M.; Reddy, A.L.M.; Yu, J.; Vajtai, R.; Ajayan, P.M. Ultrathin planar graphene supercapacitors. Nano Lett. 2011, 11, 1423-1427.

23. Liu, C.; Yu, Z.; Neff, D.; Zhamu, A.; Jang, B.Z. Graphene-based supercapacitor with an ultrahigh energy density. Nano Lett. 2010, 10, 4863-4868.

24. Liu, F.; Song, S.; Xue, D.; Zhang, H. Folded structured graphene paper for high performance electrode materials. Adv. Mater. 2012, 24, 1089-1094.

25. Wen, Z.; Wang, X.; Mao, S.; Bo, Z.; Kim, H.; Cui, S.; Lu, G.; Feng, X.; Chen, J. Crumpled nitrogen-doped graphene nanosheets with ultrahigh pore volume for high-performance supercapacitor. Adv. Mater. 2012, 24, 5610-5616.

26. Si, Y.; Samulski, E.T. Exfoliated graphene separated by platinum nanoparticles. Chem. Mater. 2008, 20, 6792-6797.

27. Huang, Z.-D.; Zhang, B.; Oh, S.-W.; Zheng, Q.-B.; Lin, X.-Y.; Yousefi, N.; Kim, J.-K. Self-assembled reduced graphene oxide/carbon nanotube thin films as electrodes for supercapacitors. J. Mater. Chem. 2012, 22, 3591-3599. 
28. Cheng, Q.; Tang, J.; Ma, J.; Zhang, H.; Shinya, N.; Qin, L.-C. Graphene and carbon nanotube composite electrodes for supercapacitors with ultra-high energy density. Phys. Chem. Chem. Phys. 2011, 13, 17615-17624.

29. Wang, Y.; Wu, Y.; Huang, Y.; Zhang, F.; Yang, X.; Ma, Y.; Chen, Y. Preventing graphene sheets from restacking for high-capacitance performance. J. Phys. Chem. C 2011, 115, 23192-23197.

30. Fan, Z.; Yan, J.; Zhi, L.; Zhang, Q.; Wei, T.; Feng, J.; Zhang, M.; Qian, W.; Wei, F. A three-dimensional carbon nanotube/graphene sandwich and its application as electrode in supercapacitors. Adv. Mater. 2010, 22, 3723-3728.

31. Xu, Z.; Li, Z.; Holt, C.M. B.; Tan, X.; Wang, H.; Amirkhiz, B.S.; Stephenson, T.; Mitlin, D. Electrochemical supercapacitor electrodes from sponge-like graphene nanoarchitectures with ultrahigh power density. J. Phys. Chem. Lett. 2012, 3, 2928-2933.

32. Yang, X.; Zhu, J.; Qiu, L.; Li, D. Bioinspired effective prevention of restacking in multilayered graphene films: towards the next generation of high-performance supercapacitors. Adv. Mater. 2011, 23, 2833-2838.

33. Kumar, N.A.; Choi, H.; Shin, Y.R.; Chang, D.W.; Dai, L. Polyaniline-grafted reduced graphene oxide for efficient electrochemical supercapacitors. ACS Nano 2012, 6, 1715-1723.

34. An, J.; Liu, J.; Zhou, Y.; Zhao, H.; Ma, Y.; Li, M.; Yu, M.; Li, S. Polyaniline-grafted graphene hybrid with amide groups and its use in supercapacitors. J. Phys. Chem. C 2012, 116, 19699-19708.

35. Wu, Q.; Xu, Y.; Yao, Z.; Liu, A.; Shi, G. Supercapacitors based on flexible graphene/polyaniline nanofiber composite films. ACS Nano 2010, 4, 1963-1970.

36. Xu, J.; Wang, K.; Zu, S.-Z.; Han, B.-H.; Wei, Z. Hierarchical nanocomposites of polyaniline nanowire arrays on graphene oxide sheets with synergistic effect for energy storage. ACS Nano 2010, 4, 5019-5026.

37. Zhang, J.; Zhao, X.S. Conducting polymers directly coated on reduced graphene oxide sheets as high-performance supercapacitor electrodes. J. Phys. Chem. C 2012, 116, 5420-5426.

38. Zhang, L.L.; Zhao, S.; Tian, X.N.; Zhao, X.S. Layered graphene oxide nanostructures with sandwiched conducting polymers as supercapacitor electrodes. Langmuir 2010, 26, 17624-17628.

39. Rakhi, R.B.; Chen, W.; Cha, D.; Alshareef, H.N. Nanostructured ternary electrodes for energy-storage applications. Adv. Energy Mater. 2012, 2, 381-389.

40. Yu, G.; Hu, L.; Liu, N.; Wang, H.; Vosgueritchian, M.; Yang, Y.; Cui, Y.; Bao, Z. Enhancing the supercapacitor performance of graphene/ $\mathrm{MnO}_{2}$ nanostructured electrodes by conductive wrapping. Nano Lett. 2011, 11, 4438-4442.

41. Yu, G.; Hu, L.; Vosgueritchian, M.; Wang, H.; Xie, X.; McDonough, J.R.; Cui, X.; Cui, Y.; Bao, Z. Solution-processed graphene/ $\mathrm{MnO}_{2}$ nanostructured textiles for high-performance electrochemical capacitors. Nano Lett. 2011, 11, 2905-2911.

42. Cheng, Y.; Lu, S.; Zhang, H.; Varanasi, C. V; Liu, J. Synergistic effects from graphene and carbon nanotubes enable flexible and robust electrodes for high-performance supercapacitors. Nano Lett. 2012, 12, 4206-4211.

43. Du, F.; Yu, D.; Dai, L.; Ganguli, S.; Varshney, V.; Roy, A.K. Preparation of tunable 3D pillared carbon nanotube - Graphene networks for high-performance capacitance. Chem. Mater. 2011, 23, 4810-4816. 
44. Yan, J.; Wei, T.; Fan, Z.; Qian, W.; Zhang, M.; Shen, X.; Wei, F. Preparation of graphene nanosheet/carbon nanotube/polyaniline composite as electrode material for supercapacitors. $J$. Power Sources 2010, 195, 3041-3045.

45. Xu, Y.; Sheng, K.; Li, C.; Shi, G. Self-assembled graphene hydrogel via a one-step hydrothermal process. ACS Nano 2010, 4, 4324-4330.

46. Niu, Z.; Chen, J.; Hng, H.H.; Ma, J.; Chen, X. A leavening strategy to prepare reduced graphene oxide foams. Adv. Mater. 2012, 24, 4144-4150.

47. Cao, X.; Shi, Y.; Shi, W.; Lu, G.; Huang, X.; Yan, Q.; Zhang, Q.; Zhang, H. Preparation of novel 3D graphene networks for supercapacitor applications. Small 2011, 7, 3163-3168.

48. Zhao, Y.; Liu, J.; Hu, Y.; Cheng, H.; Hu, C.; Jiang, C.; Jiang, L.; Cao, A.; Qu, L. Highly compression-tolerant supercapacitor based on polypyrrole-mediated graphene foam electrodes. Adv. Mater. 2013, 25, 591-595.

49. Biener, J.; Dasgupta, S.; Shao, L.; Wang, D.; Worsley, M.A; Wittstock, A.; Lee, J.R.I.; Biener, M.M.; Orme, C.A; Kucheyev, S.O.; Wood, B.C.; Willey, T.M.; Hamza, A.V; Weissmüller, J.; Hahn, H.; Baumann, T.F. Macroscopic 3D nanographene with dynamically tunable bulk properties. Adv. Mater. 2012, 24, 5083-5087.

50. Sheng, K.; Sun, Y.; Li, C.; Yuan, W.; Shi, G. Ultrahigh-rate supercapacitors based on eletrochemically reduced graphene oxide for ac line-filtering. Sci. Rep. 2012, 2, doi:10.1038/srep00247.

51. Li, J.; Ye, F.; Vaziri, S.; Muhammed, M.; Lemme, M.C.; Östling, M. Efficient inkjet printing of graphene. Adv. Mater. 2013, submitted for publication.

52. Li, J.; Ye, F.; Vaziri, S.; Muhammed, M.; Lemme, M.C.; Östling, M. A simple route towards high-concentration surfactant-free graphene dispersions. Carbon 2012, 50, 3113-3116.

53. Gao, W.; Singh, N.; Song, L.; Liu, Z.; Reddy, A.L. M.; Ci, L.; Vajtai, R.; Zhang, Q.; Wei, B.; Ajayan, P.M. Direct laser writing of micro-supercapacitors on hydrated graphite oxide films. Nat. Nanotechnol. 2011, 6, 496-500.

54. Chen, J.; Sheng, K.; Luo, P.; Li, C.; Shi, G. Graphene hydrogels deposited in nickel foams for high-rate electrochemical capacitors. Adv. Mater. 2012, 24, 4569-4573.

55. Dong, X.-C.; Xu, H.; Wang, X.-W.; Huang, Y.-X.; Chan-Park, M.B.; Zhang, H.; Wang, L.-H.; Huang, W.; Chen, P. 3D graphene-cobalt oxide electrode for high-performance supercapacitor and enzymeless glucose detection. ACS Nano 2012, 6, 3206-3213.

(C) 2013 by the authors; licensee MDPI, Basel, Switzerland. This article is an open access article distributed under the terms and conditions of the Creative Commons Attribution license (http://creativecommons.org/licenses/by/3.0/). 